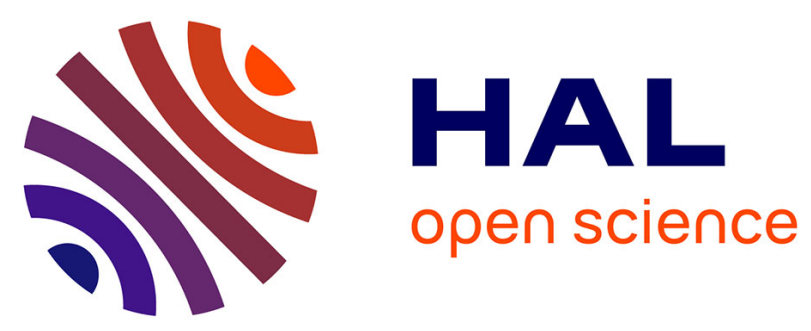

\title{
Tracking the microstructural properties of the main white matter pathways underlying speech processing in simultaneous interpreters
}

Stefan Elmer, Jürgen Hänggi, Lucía Vaquero, Guillem Olivé Cadena, Clément François, Antoni Rodríguez-Fornells

\section{To cite this version:}

Stefan Elmer, Jürgen Hänggi, Lucía Vaquero, Guillem Olivé Cadena, Clément François, et al.. Tracking the microstructural properties of the main white matter pathways underlying speech processing in simultaneous interpreters. NeuroImage, 2019, 191, pp.518-528. 10.1016/j.neuroimage.2019.02.056 . hal-02099969

\section{HAL Id: hal-02099969 https://hal.science/hal-02099969}

Submitted on 6 Nov 2020

HAL is a multi-disciplinary open access archive for the deposit and dissemination of scientific research documents, whether they are published or not. The documents may come from teaching and research institutions in France or abroad, or from public or private research centers.
L'archive ouverte pluridisciplinaire HAL, est destinée au dépôt et à la diffusion de documents scientifiques de niveau recherche, publiés ou non, émanant des établissements d'enseignement et de recherche français ou étrangers, des laboratoires publics ou privés. 


\title{
Tracking the Microstructural Properties of the Main White Matter \\ Pathways Underlying Speech Processing in Simultaneous Interpreters
}

\author{
Stefan Elmer ${ }^{\mathrm{a}, \mathrm{b} \$ \dagger}$, Jürgen Hänggi ${ }^{\mathrm{b} \dagger}$, Lucía Vaquero ${ }^{\mathrm{a}, \mathrm{f,g}}$, Guillem Olivé Cadena ${ }^{\mathrm{c}}$, Clément \\ François $^{\mathrm{a}, \mathrm{c}, \mathrm{e}, \mathrm{h}} \&$ Antoni Rodríguez-Fornells ${ }^{\mathrm{a}, \mathrm{c}, \mathrm{d}}$
}

${ }^{a}$ Cognition and Brain Plasticity Group, Bellvitge Biomedical Research Institute, L'Hospitalet de Llobregat, 08097

Barcelona, Spain

${ }^{\mathrm{b}}$ Division Neuropsychology, Department of Psychology, University of Zurich, Zurich, Switzerland

${ }^{\mathrm{c}}$ Department of Cognition, Development and Educational Psychology, Campus Bellvitge, University of Barcelona,

L’Hospitalet de Llobregat, 08097 Barcelona, Spain

${ }^{\mathrm{d}}$ Institució Catalana de Recerca i Estudis Avançats, ICREA, 08010 Barcelona, Spain

${ }^{\mathrm{e}}$ Institut de Recerca Pediàtrica Hospital Sant Joan de Déu, Barcelona, Spain

${ }^{\mathrm{f}}$ Department of cognition, development and Education Pychology, University of Barcelona, Passeig de la Vall

d'Hebron, 171, 08035, Barcelona, Spain

${ }^{\mathrm{g}}$ Laboratory of Motor learning and Neural Plasticity, Concordia University, 7141 Rue Sherbrooke West, H4B 1R6,

Montreal, QC, Canada

${ }^{\mathrm{h}}$ Aix Marseille University, CNRS, LPL, Aix-en-Provence, France

$\S$ Corresponding author

$\uparrow$ Shared first authorship

Abbreviated title: The Pathways to Language in Simultaneous Interpreters

Keywords: DTI, probabilistic and deterministic tractography, dorsal and ventral streams, arcuate fasciculus, plasticity, simultaneous interpreters

Number of words for Abstract, Introduction, and Discussion: 242/900/2918

Conflict of Interest: none declared

E-mail addresses:

SE: s.elmer@psychologie.uzh.ch

JH: j.haenggi@psychologie.uzh.ch

LV: 1ucia.vaquero.z@gmail.com

GOC: golivec95@gmail.com

CF: fclement24@hotmail.com

ARF: arfornells@gmail.com

Correspondence to:

Dr. Stefan Elmer, Institute of Psychology, Division Neuropsychology, University of Zurich, Binzmühlestrasse 14/25, 8050 Zurich, Switzerland. E-mail: s.elmer@psychologie.uzh.ch

Date: 11.02 .2019 


\section{ABSTRACT}

Due to the high linguistic and cognitive demands placed on real-time language translation, professional simultaneous interpreters (SIs) have previously been proposed to serve as a reasonable model for evaluating experience-dependent brain properties. However, currently it is still unknown whether intensive language training during adulthood might be reflected in microstructural changes in language-related white matter pathways contributing to sound-to-meaning mapping, auditory-motor integration, and verbal memory functions. Accordingly, we used a fully automated probabilistic tractography algorithm and compared the white matter microstructure of the bilateral inferior longitudinal fasciculus (ILF), uncinate fasciculus (UF), and arcuate fasciculus (AF, long and anterior segments) between professional SIs and multilingual control participants. In addition, we classically re-evaluated the three constitutional elements of the $\mathrm{AF}$ (long, anterior, and posterior segments) using a deterministic manual dissection procedure. Automated probabilistic tractography demonstrated overall reduced mean fractional anisotropy (FA) and increased radial diffusivity (RD) in SIs in the fiber tracts of the left hemisphere (LH). Furthermore, SIs exhibited reduced mean FA in the bilateral AF. However, according to manual dissection, this effect was limited to the anterior $\mathrm{AF}$ segment and accompanied by increased mean RD. Deterministic AF reconstruction also uncovered increased mean FA in the right and $\mathrm{RD}$ in the left long AF segment in SIs compared to controls. These results point to a relationship between simultaneous interpreting and white matter organization of pathways underlying speech and language processing in the language-dominant LH as well as of the $\mathrm{AF}$. 


\section{INTRODUCTION}

Numerous studies have demonstrated that both short- and long-term regimens of effortful activities characterized by a high amount of deliberate practice lead to functional and structural brain changes (Bezzola et al., 2011; Havas et al., 2017; Hyde et al., 2009; Palomar-Garcia et al., 2017). Among such activities, simultaneous interpreting has been proposed to serve as a reasonable model for evaluating experience-dependent brain plasticity in adulthood (Elmer et al., 2014; Elmer et al., 2010a; Hervais-Adelman et al., 2017), functional and anatomical correlates of multilingual speech processing (Elmer et al., 2010b; Proverbio et al., 2004; Rinne et al., 2000), and language-related cognitive control mechanisms (Becker et al., 2016; Elmer, 2016; Hervais-Adelman et al., 2015). This perspective is anchored on the wide range of parallel linguistic and cognitive processes involved in simultaneous interpreting. In fact, this activity requires maintaining the source information in short-term and working memory, accessing phonological and semantic representations across languages, switching between languages, monitoring the languages in use, and producing the target language (Elmer, 2016; Kroll, 2005).

Previous neuroimaging studies have documented increased brain activity during interpreting, compared to shadowing, in the left ventral prefrontal cortex, inferior parietal lobule (IPL), and distributed temporal areas (Elmer, 2016; Hervais-Adelman et al., 2015; Klein et al., 2006; Price et al., 1999; Rinne et al., 2000) which contribute to phonetic processing (Ruff et al., 2003; Turkeltaub and Coslett, 2010), phonological categorization (Joanisse et al., 2007; Turkeltaub and Coslett, 2010), and lexicalsemantic operations (Elmer et al., 2010b; Obleser et al., 2008). Furthermore, the high 
cognitive and linguistic demands placed on simultaneous interpreting, especially in terms of language switching, working memory, inhibition, and monitoring functions, have been associated with training-related structural peculiarities in the ventral and dorsal parts of the prefrontal cortex, IPL, anterior cingulate cortex (ACC), caudate nuclei, and posterior superior temporal (pST) areas (Becker et al., 2016; Elmer et al., 2014; Elmer et al., 2010a; Hervais-Adelman et al., 2017; Van de Putte et al., 2018).

Notwithstanding that a fairly complete description of the main white matter infrastructure underlying language processing has been provided (Friederici, 2009; Hickok and Poeppel, 2007; Rauschecker and Scott, 2009; Saur et al., 2008), currently only little is known about the relationships between interpreting training and these language-related pathways (Elmer et al., 2010a; Van de Putte et al., 2018). In the context of a longitudinal study, Van de Putte and colleagues (Van de Putte et al., 2018) used a network-based statistical approach and compared structural connectivity between interpreting students and a matched group of translators before and after nine months of training. As a main result, the authors revealed significant training-related increased structural connectivity in interpreting students in two networks supporting cognitive control mechanisms. The first network was reflected by increased connectivity between frontal brain regions and the basal ganglia, whereas the second one consisted of higher structural connectivity between the supplementary motor area, postcentral gyrus, superior frontal gyrus, middle temporal pole, amygdala, vermis of the cerebellum and the parietal lobe. In a second cross-sectional anatomical study, Elmer and colleagues (Elmer et al., 2010a) compared fractional anisotropy (FA) between professional SIs and multilingual control participants using tract-based spatial statistics. This study revealed overall lower FA values in SIs compared to controls in brain regions supporting 
sensory-to-motor coupling mechanisms, articulation, interhemispheric transfer and cognitive control, including the forceps minor and major of the corpus callosum, left anterior insula, head of the right caudate, left cingulum and right IPL.

The inferior longitudinal fasciculus (ILF) and the uncinate fasciculus (UF) are part of the ventral stream, and contribute to sound-to-meaning mapping and auditory object recognition (Catani et al., 2003; Friederici, 2009; Saur et al., 2008). Otherwise, the arcuate fasciculus (AF) forms the main infrastructure of the dorsal stream and connects pST regions with Broca's area (long segment), the IPL with Broca's area (anterior segment), and pST regions with the IPL (posterior segment) (Catani et al., 2007; Catani et al., 2005). The long segment facilitates sound-to-articulation transformations (Hickok and Poeppel, 2007; Liberman and Mattingly, 1985; Lopez-Barroso et al., 2013), phonological processes (Vandermosten et al., 2012), prosody (Sammler et al., 2015), and working memory functions (Meyer et al., 2014). Furthermore, although the functional accounts of the AF are far from unequivocal, the posterior segment is possibly involved in phonological processing and working memory, whereas the anterior segment mediates higher-order language functions and verbal memory (Friederici, 2009, 2012; Rilling et al., 2008). In the present anatomical study, we compared the microstructural properties of the main white matter pathways underlying speech and language processing, namely the bilateral AF, ILF, and UF, between professional SIs and multilingual control participants. Overall, based on the dominance of the $\mathrm{LH}$ for language processing in general and simultaneous interpreting in particular (Corballis, 2010; Rinne et al., 2000), we hypothesized that the main microstructural between-group differences will be restricted to this hemisphere. In addition, due to the high demands placed on auditory-motor integration and working memory in SIs (Elmer 
et al., 2010a; Elmer and Kuhnis, 2016), we predicted between-group differences in the left long and anterior AF segments. Finally, for reasons of completeness and based on our previous work showing white matter changes in the forceps minor and major of the corpus callosum in SIs compared to controls (Elmer et al., 2010a), we also evaluated these two fiber tracts. However, since the corpus callosum is not a pathway usually characterized or studied in language processing, these additional results were implemented as supplementary material.

\section{MATERIALS \& METHODS}

\subsection{Participants}

In the present work, we analyzed the main language-related white matter pathways in 15 professional SIs $(11$ women, mean age $=38.3$ years, standard deviation $(\mathrm{SD})=5.74$ years; mean number of years of interpreting experience $=9.82, \mathrm{SD}=6.53$, the information of one SI is missing) and 16 control participants (11 women, mean age = 32.2 years, $\mathrm{SD}=7.49$ years) with fluent multilingual speech competence (Table 1$)$. Notwithstanding that gender and education (i.e., university degree or advanced university students) were matched, the two groups differed in age (t-test, $\mathrm{t}_{(29)}=-2.542, \mathrm{p}$ $=.017)$. Accordingly, this variable was used as a covariate of no interest in the statistical analyses. All participants were right-handed (Annett, 1970), native German speakers, and did not report a history of neurological, psychiatric or audiological disorders. None of the participants was simultaneous or early bilingual, or started learning their foreign languages before enrolling in school. The study was approved by the local ethics committee (i.e.,Cantonal Ethics Commission Zurich) and conducted in accordance with the declaration of Helsinki. Eleven SIs and 11 control participants had already been included in a previous study (Elmer et al., 2010a). However, in the present 
work we addressed a new research topic that is completely unrelated to that of our previous work.

\begin{tabular}{|c|c|c|c|c|c|}
\hline Group & Participant & Age & Gender & Number of experience years & Spoken languages \\
\hline \multirow[t]{15}{*}{ SI } & 1 & 36 & $\mathrm{f}$ & 2 & GER, POR, ENG \\
\hline & 2 & 46 & $\mathrm{f}$ & 22 & GER, ENG, FR, IT, SP \\
\hline & 3 & 48 & $f$ & 15 & GER, IT, FR \\
\hline & 4 & 32 & $f$ & 4.5 & GER, SP, ENG, FR, POR \\
\hline & 5 & 41 & $f$ & 10 & GER, FR \\
\hline & 6 & 37 & $\mathrm{~m}$ & 11 & GER, EN, FR, SP \\
\hline & 7 & 34 & $\mathrm{~m}$ & 5 & GER, ENG, FR \\
\hline & 8 & 39 & $\mathrm{~m}$ & 8 & GER, ENG, FR \\
\hline & 9 & 36 & $\mathrm{f}$ & 9 & GER, ENG \\
\hline & 10 & 35 & $f$ & 9 & GER, FR \\
\hline & 11 & 39 & f & & GER, ENG, FR, SP \\
\hline & 12 & 33 & $f$ & 2 & GER, IT, FR, ENG, SP \\
\hline & 13 & 38 & $f$ & 14 & GER, IT, FR, ENG \\
\hline & 14 & 50 & $f$ & 22 & GER, IT, FR, ENG \\
\hline & 15 & 31 & $\mathrm{~m}$ & 4 & IT; ENG; FR; GER \\
\hline \multirow[t]{16}{*}{ CON } & 1 & 26 & $\mathrm{~m}$ & & GER, ENG \\
\hline & 2 & 27 & $f$ & & GER; POR; ENG, DU \\
\hline & 3 & 26 & $f$ & & GER; ENG; FR \\
\hline & 4 & 27 & $f$ & & GER, ENG \\
\hline & 5 & 34 & $\mathrm{~m}$ & & GER, FR, ENG \\
\hline & 6 & 30 & $\mathrm{~m}$ & & SP, GER, ENG \\
\hline & 7 & 28 & $\mathrm{~m}$ & & FR, GER, ENG \\
\hline & 8 & 27 & $f$ & & GER, ENG, FR \\
\hline & 9 & 26 & $f$ & & GER, ENG, FR \\
\hline & 10 & 25 & $f$ & & GER, ENG, FR \\
\hline & 11 & 25 & $\mathrm{f}$ & & GER, ENG, FR \\
\hline & 12 & 46 & f & & GER, ENG, FR \\
\hline & 13 & 42 & $\mathrm{f}$ & & GER, ENG, FR \\
\hline & 14 & 39 & f & & GER, ENG, FR \\
\hline & 15 & 42 & $f$ & & GER, ENG, FR \\
\hline & 16 & 43 & $\mathrm{~m}$ & & GER, ENG, FR \\
\hline
\end{tabular}

Table 1. Biographical data of the participants. GER = German; FR = French; ENG = English; IT = Italian; SP = Spanish; POR = Portuguese; DU = Dutch. SI = simultaneous interpreters; $\mathrm{CON}=$ multilingual control participants.

- Please insert Table 1 about here - 


\subsection{Imaging data acquisition}

Magnetic resonance imaging (MRI) scans were acquired on a 3.0 Tesla Philips Achieva whole body scanner (Philips Medical Systems, Best, The Netherlands) equipped with a transmit-receive body coil and a commercial eight-element sensitivity encoding (SENSE) head coil array. Scanning was performed at the University Hospital of Zurich (Switzerland) and SIs and control participants were measured in an interleaved manner. Due to technical problems during DTI data collection, two SIs and one control participant were excluded from the analyses. A diffusion-weighted echo-planar imaging (EPI) sequence (spin echo) was used to obtain diffusion-weighted scans with a measured spatial resolution of $2.08 \times 2.08 \times 2.0 \mathrm{~mm}$ (acquisition matrix $96 \times 96$ pixels, 50 slices) and a reconstructed resolution of $1.56 \times 1.56 \times 2.0 \mathrm{~mm}$ (reconstructed matrix 128 x 128 pixels, 50 slices). Further imaging parameters were: field of view, FOV $=200$ x $200 \mathrm{~mm}^{2}$; echo time, $\mathrm{TE}=50 \mathrm{~ms}$; repetition time, $\mathrm{TR}=10,166 \mathrm{~ms}$; flip angle $=90^{\circ}$; SENSE factor, $\mathrm{R}=2.1$; and $\mathrm{b}$ value $=1,000 \mathrm{~s} / \mathrm{mm}^{2}$. We used the Philips standard diffusion gradient mode ("medium") that comprises 16 diffusion directions (15 noncollinear diffusion directions and one non-diffusion weighted reference volume). Total acquisition time was about 8 minutes.

A volumetric 3D T1-weighted gradient echo sequence (TFE, turbo field echo) image was measured with a spatial resolution of $1 \times 1 \times 1.5 \mathrm{~mm}^{3}$ (acquisition matrix $224 \times 224$ pixels, 90 slices) and reconstructed to a resolution of $0.86 \times 0.86 \times 0.75 \mathrm{~mm}^{3}$ (reconstructed matrix 256 x 256 pixels, 180 slices). Further imaging parameters were: $\mathrm{FOV}=220 \times 220 \mathrm{~mm}^{2}, \mathrm{TE}=2.3 \mathrm{msec}, \mathrm{TR}=20 \mathrm{msec}$, flip angle $=20^{\circ}$. 


\subsection{Automated probabilistic tractography analyses}

T1-weighted images were processed using the FreeSurfer software suite version 6.0.0 (http://surfer.nmr.mgh.harvard.edu/fswiki) that was originally designed to perform surface-based morphometry. This software was used to obtain the seed points (distributions on the neighboring anatomical structures) needed for fiber tractography. The preprocessing steps of the T1-weighted MRI scans are described in more details elsewhere (http://surfer.nmr.mgh.harvard.edu/fswiki/FreeSurferMethodsCitation).

DTI data were processed with the tracts constrained by underlying anatomy (TRACULA) toolbox (https://surfer.nmr.mgh.harvard.edu/fswiki/Tracula). The TRACULA toolbox, which relies on a global probabilistic tractography approach with anatomical priors, was used for the automated reconstruction of a set of 18 major white matter pathways. The TRACULA toolbox is based on software of the FSL tool (FMRIB software library; $\quad$ version $\quad 5.0 .6 ; \quad \underline{\text { http://www.fmrib.ox.ac.uk/fsl/) }}$ (https://www.ncbi.nlm.nih.gov/pubmed/15501092) and is implemented in the FDT $\begin{array}{llll}\text { toolkit } & \text { (FMRIB } & \text { diffusion } & \text { toolbox; }\end{array}$ (https://www.ncbi.nlm.nih.gov/pubmed/14587019). Prior distributions on the neighboring anatomical structures of each pathway are derived from an atlas, and combined with the FreeSurfer cortical parcellation scheme and subcortical segmentation of the participants to constrain the tractography solutions (Yendiki et al., 2011; Yendiki et al., 2016).

The processing steps of the DTI data included (1) eddy current and head movement correction (FSL's eddy tool), (2) intra-subject registration (individual diffusionweighted to individual T1-weighted MRI scan), (3) inter-subject registration (individual 
T1-weighted to a common template space), (4) creation of cortical and white-matter masks from FreeSurfer reconstructions, (5) diffusion tensor fitting, (6) computation of anatomical priors for white-matter pathways from the TRACULA atlas. Furthermore, (7) TRACULA uses the ball-and-stick model of diffusion using FSL's bedpostX tool (https://www.ncbi.nlm.nih.gov/pubmed/17070705) by estimating probability distributions of the parameters of this model at every voxel. (8) Reconstruction of white-matter pathways using FSL's probtrackX tool (https://www.ncbi.nlm.nih.gov/pubmed/17070705) by simultaneously fitting the shape of each pathway to the results of the ball-and-stick model of diffusion from above and to the prior knowledge of the pathway anatomy given by the set of manually labeled training subjects in the TRACULA atlas. More details about the preprocessing stages can be found in the original methodological publications (Yendiki et al., 2011). Since tract reconstructions were constrained by underlying anatomy, namely by numerous brain regions and not just by a seed and a target region, we do not present these regions in a figure.

Based on the fact that the present study exclusively focused on language-related pathways, we only evaluated the AF (long and anterior segments), ILF, and UF (Friederici, 2009; Saur et al., 2008). Furthermore, as supplementary analyses, we also evaluated the forceps major and minor. It is important to mention that the TRACULA toolbox only enables to reconstruct the temporal (SLFt) and parietal (SLFp) bundles of the superior longitudinal fasciculus (SLF) that roughly correspond to the long and anterior AF segments, respectively (Catani et al., 2005; Catani and Mesulam, 2008). Mean FA and RD values were extracted from the bilateral AF (long and anterior segments), ILF, and UF, and compared between the two groups. For the supplementary 
analyses, we also evaluated mean FA and RD in the forceps minor and major. Figure 1 provides automated probabilistic tractography reconstructions of the four tracts of interest constituting the language-related network in three SIs and three control participants.
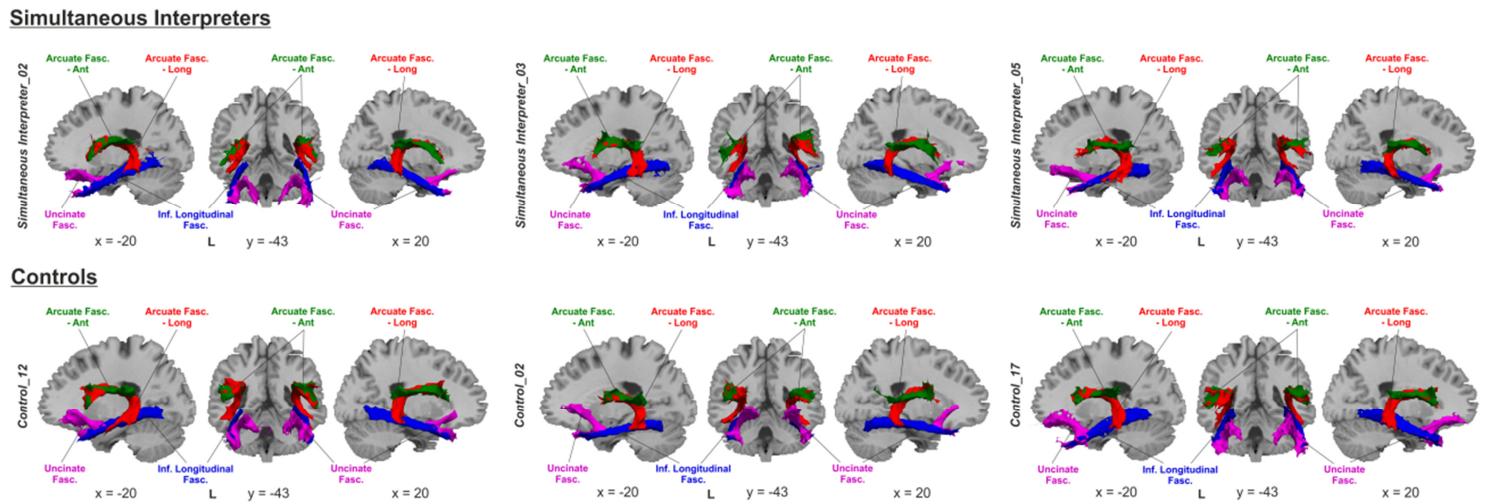

Figure 1. Automated probabilistic tractography reconstructions of the four languagerelated tracts in three simultaneous interpreters (upper row) and three control participants (lower row). Green $=$ anterior segment of the Arcuate Fasciculus, red $=$ long segment of the Arcuate Fasciculus, blue = Inferior Longitudinal Fasciculus, magenta $=$ Uncinate Fasciculus Abbreviations: Ant, anterior; Fasc., fasciculus; Inf., inferior; L, left; $x, y, z$, Montreal Neurological Institute space coordinates of the background slices.

- Please insert Figure 1 about here -

\subsection{Manual deterministic tractography analyses}

In the present work, we additionally manually dissected the AF in both hemispheres. This complementary approach does not only enable a more meticulous segmentation of the three AF segments (Catani et al., 2005; Catani and Mesulam, 2008), but also the comparability of probabilistic and deterministic tractography data. Since the manual dissection procedure used was the same as the one previously reported by Vaquero and 
colleagues (Vaquero et al., 2018; Vaquero et al., 2017), in the following paragraph we literally reiterate the description of this procedure.

"The three segments of the AF were dissected by GOC and revised by LV using three main manually defined ROIs as described in previous studies (Catani et al., 2007; Catani et al., 2005; Lopez-Barroso et al., 2013). Specifically, a first ROI was delineated in the coronal view, anterior to the central sulcus, encompassing the fibers going to the inferior frontal gyrus (IFG, including Broca's area; Brodmann's areas 44 and 45, and parts of the medial frontal gyrus). Then, in the axial view, a second ROI was depicted covering the WM underlying the medial and superior temporal gyrus (STG, embracing the fibers traveling to Wernicke's territory; Brodmann's areas 22, 41 and 42). Finally, a third ROI was drawn on the sagittal view, covering supramarginal and angular gyri and encompassing the fibers traveling to Geschwind's territory (Brodmann's areas 39 and 40). These ROIs were combined to encompass the three rami of the AF: the long (between inferior frontal gyrus-Broca's area and superior temporal gyrus-Wernicke's area), the anterior (linking inferior frontal gyrus-Broca's area and Geschwind's territory) and the posterior (connecting Wernicke's and Geschwind's territories) segments. Artefactual fibers were removed using exclusion ROIs. Figure 2 provides a manual dissection example in three SIs and three control participants." 


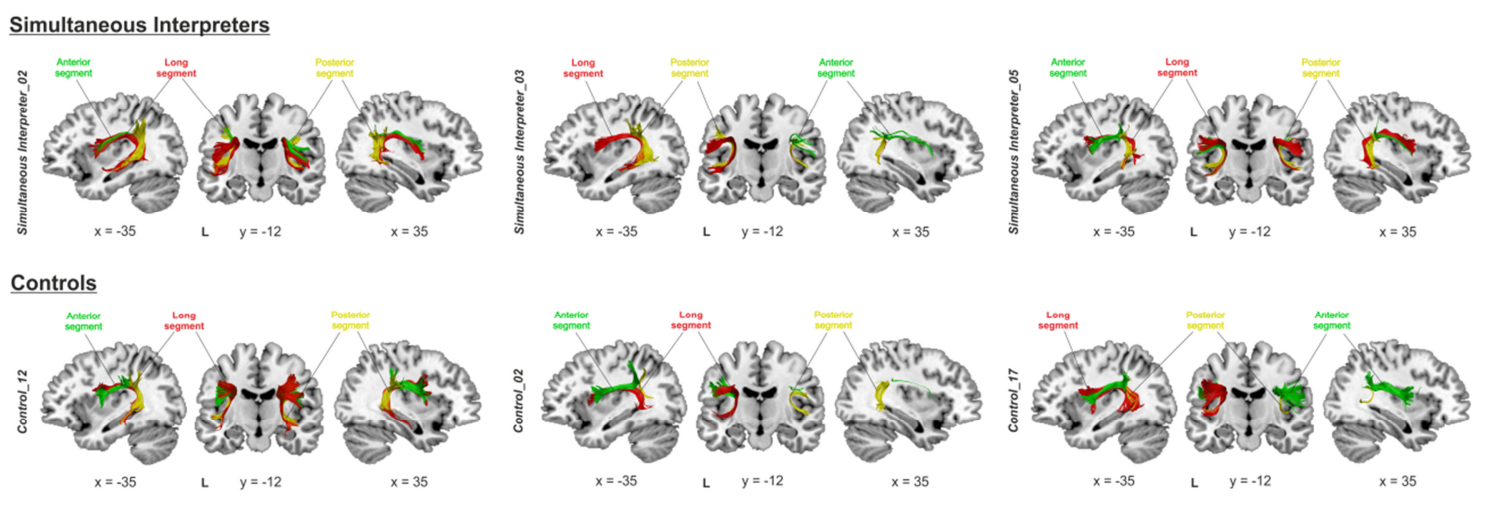

Figure 2. Manual deterministic tractography reconstructions of the three segments of the arcuate fasciculus (AF) in three simultaneous interpreters (upper row) and three control participants (lower row). Green $=$ anterior segment of the Arcuate Fasciculus, red $=$ long segment of the Arcuate Fasciculus, yellow $=$ posterior segment of the Arcuate Fasciculus. Abbreviations: $\mathrm{L}=\mathrm{left} ; \mathrm{x}, \mathrm{y}, \mathrm{z}=$ Montreal Neurological Institute space coordinates of the background slices.

- Please insert Figure 2 about here -

\subsection{Statistical analyses}

The biographical data (i.e., age and number of years of interpreting experience), FA, and $\mathrm{RD}$ (probabilistic and deterministic tractography) were tested for Gaussian distribution by using the Kolmogorov-Smirnov test. According to Gaussian data distribution $(p>.05)$, all statistical analyses were performed using parametric statistics implemented in the IBM SPSS Statistics 20 software (SPSS, an IBM company, Armonk, New York, USA). Mean FA and RD values originating from probabilistic and deterministic tractography were evaluated separately by means of analyses of covariance (ANCOVA, repeated measurements, age as covariate). In particular, the probabilistic data were subjected to a $2 \times 4 \times 2$ ANCOVA with the between-subject 
factor "group" (SIs and controls) and the within-subject factors "tract" (ILF, UF, long AF segment, and anterior AF segment) and "hemisphere" (LH and $\mathrm{RH}$ ). Otherwise, since single AF segments could not be manually reconstructed in some participants and missing values in the ANCOVA will result in list-wise deletion of individuals, the deterministic data were evaluated by means of separate ANCOVAs (repeated measurements, age as covariate) for $\mathrm{FA}$ and $\mathrm{RD}$ values and the three $\mathrm{AF}$ segments (long, anterior, and posterior). The three ANCOVA analyses were corrected for multiple comparisons (separately for FA and RD values) using a Bonferroni procedure that takes into account the mean correlation between the three AF segments in both hemispheres (Uitenbroek, 1997). Such a less conservative correction procedure is justified by the fact that correcting for multiple tests assumes independence between the different variables tested. Accordingly, common Bonferroni adjustment is used if there is no dependence (i.e., no or low correlation) between the outcome variables. However, if the outcome variables are dependent (i.e., highly correlated) a common Bonferroni adjustment would be too conservative. For the evaluation of the three segments of the AF (i.e., deterministic tractography) by means of separate ANCOVAs (i.e., three analyses for FA and three for $\mathrm{RD}$ values) we adopted this less conservative correction procedure. According to a full Bonferroni adjustment, the corrected $\mathrm{p}$ value for three comparisons would correspond to $.05 / 3=.16$. However, since the FA and RD values of the three AF segments are highly correlated we calculated the mean correlation of all segments. Accordingly, we computed a correlation matrix (3 AF segments $\mathrm{x} 2$ hemispheres) and averaged the correlation coefficients (i.e., absolute value) of all comparisons. Afterwards, we adjusted the $\mathrm{p}$ values by taking into account the mean correlation between all segments of the right and left AF using the Bonferroni procedure published 
on the SISA website (http://www.quantitativeskills.com/sisa/calculations/bonfer.htm) (Uitenbroek, 1997).

For all statistical analyses, significant interaction effects were further inspected by means of post-hoc t-tests. Furthermore, "group $\mathrm{x}$ hemisphere" interactions (FA and RD) were analyzed by computing the laterality index [i.e., Laterality Index, $\mathrm{LI}=(\mathrm{LH}-\mathrm{RH}) /$ $(\mathrm{LH}+\mathrm{RH})]$ and testing it against zero (i.e., no asymmetry) within the two groups. Moreover, in order to test relationships between microstructural white matter properties and interpreting experience, main and interaction effects involving the group factor were further inspected using correlative analyses. According to the probabilistic tractography results, mean FA and RD values of all four lefthemispheric tracts as well as FA in the bilateral long and anterior AF segments were correlated with the number of years of interpreting experience. In a similar way, based on the deterministic tractography results, we correlated mean FA and $R D$ values in the bilateral anterior AF segment as well as FA values in the right and RD values in the left long AF segment with the number of years of interpreting experience. All correlative analyses were computed according to Pearson's r (twotailed, the number of years of experience of one SI is missing), and Bonferronicorrected separately for FA and RD values as well as for probabilistic and deterministic data by taking into account the mean correlation between the variables of interest (Uitenbroek, 1997). Finally, to compare the probabilistic and deterministic data, we also correlated mean $\mathrm{FA}$ and $\mathrm{RD}$ of the entire $\mathrm{AF}$, long $\mathrm{AF}$ segment, and anterior AF segment between the two methods according to Pearson's $r$ (two-tailed, Bonferroni-corrected p value for 6 correlations computed with FA and RD 
values $=.0083$ ). Motion parameters during DTI scanning were compared between the two groups using nonparametric Mann-Whitney U-tests.

\section{RESULTS}

\subsection{Motion parameters}

Average translation (mean/SD: $1.003 / 0.241 \mathrm{~mm}$ for controls and $1.026 / 0.333 \mathrm{~mm}$ for SIs) and rotation $\left(0.0065 / 0.0030^{\circ}\right.$ for controls and $0.0067 / 0.0045^{\circ}$ for SIs) during DTI data acquisition did not significantly differ between the two groups $(U=128, p=0.57$ and $U=132, p=0.67$, respectively). Therefore, we did not use motion parameters as covariates of no interest in our statistical models.

\subsection{Automated probabilistic tractography: FA values}

The $2 \times 4 \times 2$ ANCOVA (age as covariate) with the between-subjects factor "group" and the within-subject factors "tract" (i.e., AF long, AF anterior, ILF, and UF) and "hemisphere" (LH and $\mathrm{RH})$ revealed a main effects of "hemisphere" $\left(\mathrm{F}_{(1,28)}=7.838, \mathrm{p}\right.$ $=.009)$ as well as significant "group $\mathrm{x}$ hemisphere" $\left(\mathrm{F}_{(1,28)}=5.798, \mathrm{p}=.023\right)$ and "group $x$ tract" $\left(\mathrm{F}_{(2,60)}=3.45, \mathrm{p}=.020\right)$ interaction effects (Figure 3A). The main effect of "hemisphere" was related to increased FA values in the RH compared to the LH (mean $\mathrm{RH}=0.455919$; mean $\mathrm{LH}=0.442052$ ). Otherwise, the "group $\mathrm{x}$ hemisphere" interaction was reflected by increased FA values in the LH in controls compared to SIs $\left(\mathrm{LH}: \mathrm{t}_{(29)}=2.911, \mathrm{p}=.007 ; \mathrm{RH}: \mathrm{t}_{(29)}=1.004, \mathrm{p}=.324\right.$, Figure $\left.3 \mathrm{C}\right)$, whereas the "group $\mathrm{x}$ tract" interaction originated from increased FA values in the long and anterior AF segments in controls compared to SIs (AF long: $t_{(29)}=3.436, p=.002$; AF anterior: $t_{(29)}$ $\left.=3.826, \mathrm{p}=.001 ; \mathrm{ILF}: \mathrm{t}_{(29)}=1.884, \mathrm{p}=.070 ; \mathrm{UF}: \mathrm{t}_{(29)}=.225, \mathrm{p}=.824\right)$. Finally, based on the significant "group $\mathrm{x}$ hemisphere" interaction, we tested the LI (mean FA of all 
tracts) against zero within the two groups. These two additional analyses revealed a right-hemispheric FA asymmetry in SIs $\left(\mathrm{t}_{(14)}=-7.213, \mathrm{p}<.001\right.$, FA RH $>$ LH), whereas the controls did not differ from a symmetric distribution $\left(\mathrm{t}_{(15)}=-1.191, \mathrm{p}=.252\right.$, Figure 3E).

\subsection{Automated probabilistic tractography: RD values}

The $2 \times 4 \times 2$ ANCOVA (age as covariate) with the between-subject factor "group" and the within-subject factors "tract" (i.e., AF long, AF anterior, ILF, and UF) and "hemisphere" (LH and RH) yielded a main effect of "hemisphere" $\left(\mathrm{F}_{(1,28)}=9.552, \mathrm{p}=\right.$ $.004)$ as well as a significant "group $\mathrm{x}$ hemisphere" interaction $\left(\mathrm{F}_{(1,28)}=6.132, \mathrm{p}=.020\right.$, Figure 3B). The main effect of "hemisphere" originated from increased RD values in the $\mathrm{LH}$ compared to the $\mathrm{RH}$ (mean $\mathrm{LH}=0.000565$, mean $\mathrm{RH}=0.000549$ ), whereas the "group $\mathrm{x}$ hemisphere" interaction was driven by reduced RD values in the LH in controls compared to SIs $\left(\mathrm{LH}: \mathrm{t}_{(29)}=-2.738, \mathrm{p}=.010\right.$; $\mathrm{RH}: \mathrm{t}_{(29)}=-.621, \mathrm{p}=.539$, Figure 3D). Finally, according to the significant "group $\mathrm{x}$ hemisphere" interaction, we tested the LI (mean RD of all tracts) against zero within the two groups. These additional analyses revealed a LH RD asymmetry in SIs $\left(\mathrm{t}_{(14)}=6.084, \mathrm{p}<.001, \mathrm{RD} \mathrm{LH}>\mathrm{RH}\right)$, whereas the controls showed a symmetric distribution $\left(\mathrm{t}_{(15)}=1.048, \mathrm{p}=.311\right.$, Figure $3 \mathrm{~F})$. 
A)

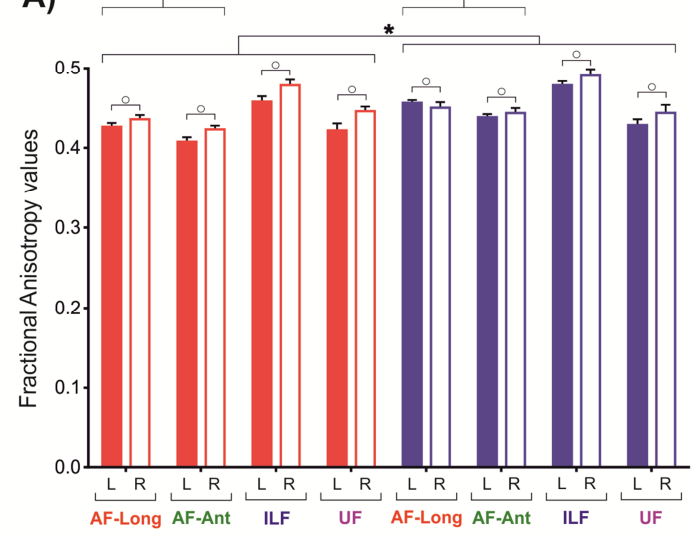

C)

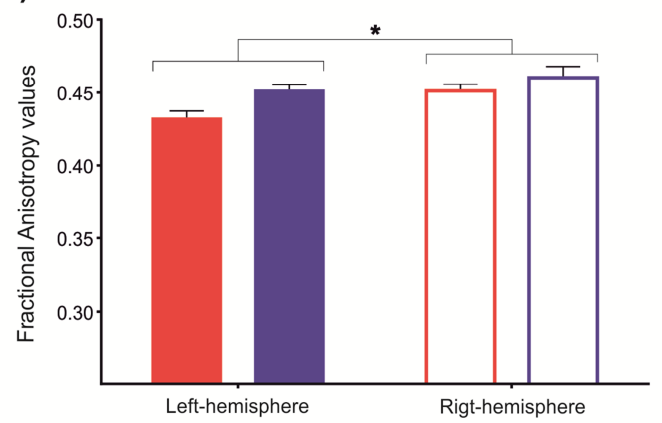

E)

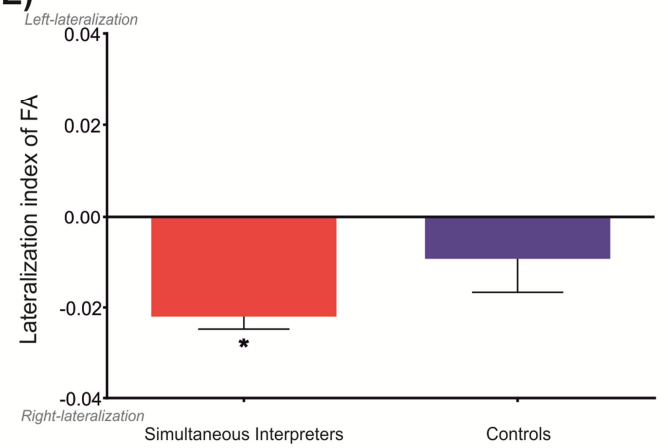

B)

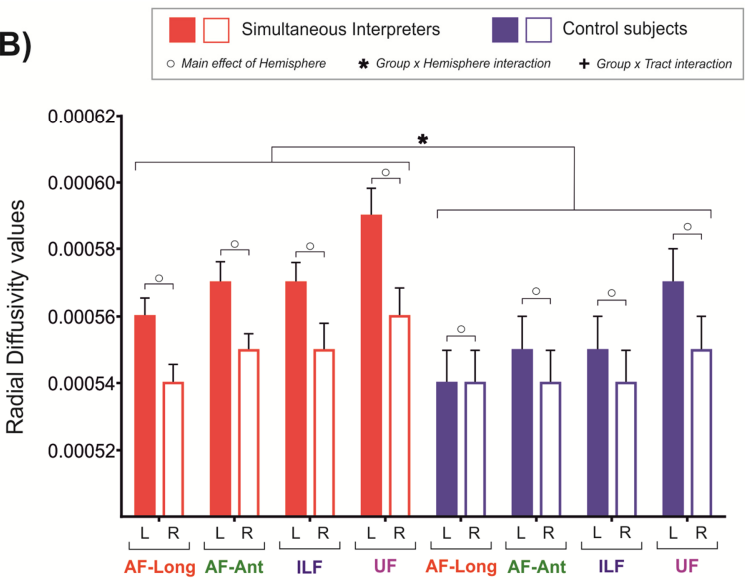

D)

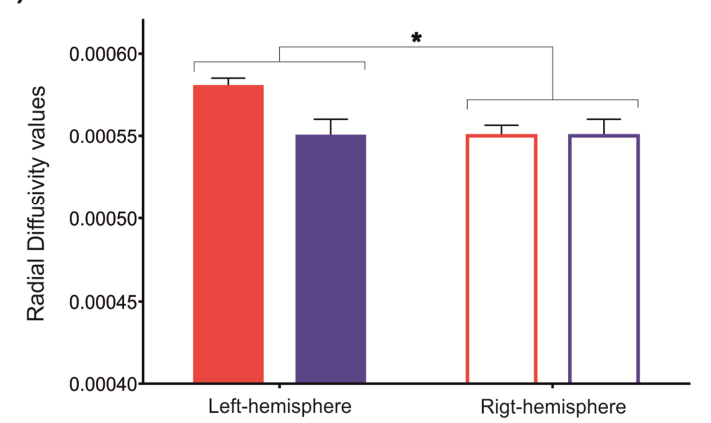

F)

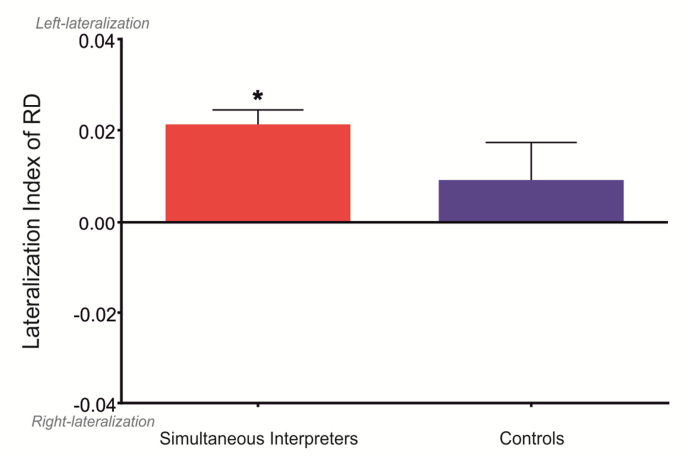

Figure 3. Significant results of the automated probabilistic tractography procedure: (A) FA, "group $\mathrm{x}$ hemisphere" and "group $\mathrm{x}$ tract" interactions; (B) RD, "group $\mathrm{x}$ hemisphere" interaction; (C) FA, "group x hemisphere” interaction; (D) RD, "group x hemisphere" interaction; (E) mean FA of all tracts, LI; (F) mean RD of all tracts, LI. Red $=$ SIs, blue $=$ controls, colored bars $=\mathrm{LH}$, bordered bars $=\mathrm{RH}$. Abbreviations: AFLong, long segment of the Arcuate Fasciculus; AF-Ant, anterior segment of the Arcuate Fasciculus; ILF, inferior Longitudinal Fasciculus; UF, Uncinate Fasciculus. 


\subsection{Manual deterministic tractography: FA values}

The left long AF could be successfully dissected in each individual participant. However, in some individuals, we were not able to reconstruct the left anterior ( $3 \mathrm{C}$ and 4 SIs), left posterior (2 SIs), right long (9 C and 4 SIs), right anterior (1 C), and right posterior (3 C and $3 \mathrm{SIs}$ ) AF segments. Accordingly, in order to overcome the problem of list-wise deletions of participants in the omnibus analyses resulting in small sample sizes, the three AF segments were evaluated by means of three separate 2 (groups) $\mathrm{x} 2$ (hemispheres) ANCOVAs (age as covariate). The three ANCOVAs were Bonferronicorrected by taking into account the mean correlation between the FA values of the three LH and RH tracts (see methods, corrected $p$ value $=.027$ ). The evaluation of the long AF segment revealed a "group $x$ hemisphere" interaction $\left(\mathrm{F}_{(1,15)}=7.516, \mathrm{p}=.015\right)$, whereas the analysis of the anterior segment yielded a main effect of "group" $\left(F_{(1,20)}=\right.$ 5.912, $\mathrm{p}=.025)$. Otherwise, we did not reveal significant main effects or interactions in the posterior AF segment (Figure 4A). The "group $\mathrm{x}$ hemisphere" interaction in the long AF segment originated from increased FA values in SIs compared to controls in the RH $\left(\mathrm{RH} \mathrm{t}_{(16)}=-2.140, \mathrm{p}=.048 ; \mathrm{LH} \mathrm{t}_{(29)}=1.513, \mathrm{p}=.141\right)$. In addition, the main effect of "group" in the anterior AF segment was related to overall smaller mean FA values in SIs compared to controls (mean SIs $=0.4222$; mean controls $=0.4490)$. Finally, based on the significant "group $\mathrm{x}$ hemisphere" interaction we revealed in the long AF segment, we additionally tested the LI against zero within the two groups. These additional two analyses revealed a RH FA asymmetry in SIs $\left(\mathrm{t}_{(10)}=-4.880, \mathrm{p}=.001, \mathrm{RH}\right.$ $>$ LH), whereas the controls showed a symmetric distribution $\left(\mathrm{t}_{(6)}=.708, \mathrm{p}=.506\right.$, Figure 4C). 


\subsection{Manual deterministic tractography: RD values}

Due to reconstruction problems that will result in list-wise deletions of participants in the omnibus analyses, we performed separate 2 (groups) x 2 (hemispheres) ANCOVAs (age as covariate) for each AF segment. The three ANCOVAs were Bonferronicorrected by taking into account the mean correlation between the mean $\mathrm{RD}$ values of the three LH and RH tracts (see methods, corrected $\mathrm{p}$ value $=.031$ ). The evaluation of the long AF segment revealed a significant "group $\mathrm{x}$ hemisphere" interaction $\left(\mathrm{F}_{(1,15)}=\right.$ $10.976, \mathrm{p}=.005)$ that originated from increased mean RD values in SIs in the LH (LH $\left.\mathrm{t}_{(29)}=-2.326, \mathrm{p}=.027 ; \mathrm{RH} \mathrm{t} \mathrm{t}_{(16)}=1.119, \mathrm{p}=.280\right)$. The analysis of the anterior AF segment yielded main effects of "group" $\left(\mathrm{F}_{(1,21)}=5.840, \mathrm{p}=.025\right)$ and "hemisphere" $\left(\mathrm{F}_{(1,21)}=5.858, \mathrm{p}=.025\right)$, whereas we did not reveal significant results in the posterior AF segment (Figure 4B). The main effect of "group" in the anterior AF segment was related to increased mean RD values in SIs compared to controls (mean SIs $=0.000558$; mean controls $=0.000519)$. Otherwise, the main effect of "hemisphere" in the same AF segment originated from increased RD values in the LH compared to the RH (mean RD $\mathrm{LH}=0.000561$; mean $\mathrm{RD} \mathrm{RH}=0.000513$ ). Finally, based on the significant "group $\mathrm{x}$ hemisphere" interaction effect we revealed in the long AF segment, we additionally tested the LI against zero within the two groups. These additional two analyses revealed a LH RD asymmetry in SIs $\left(\mathrm{t}_{(10)}=5.935, \mathrm{p}<.001, \mathrm{LH}>\mathrm{RH}\right)$ but not in the control $\operatorname{group}\left(\mathrm{t}_{(6)}=-.583, \mathrm{p}=.581\right.$, Figure 4D). 
A)

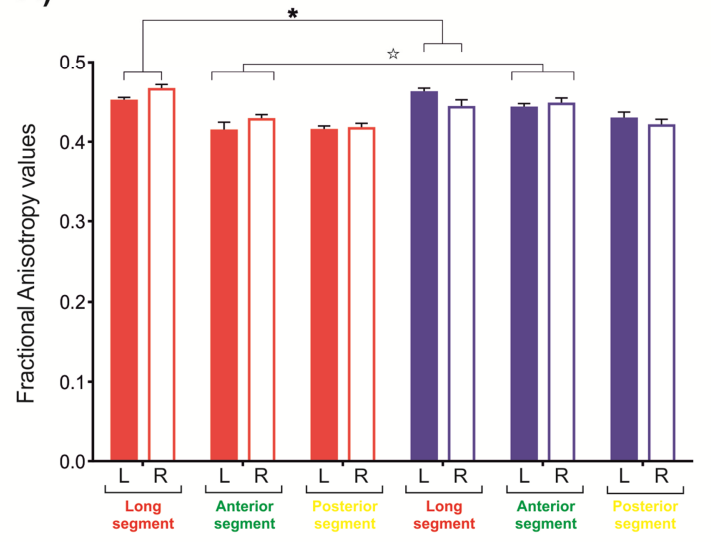

C)

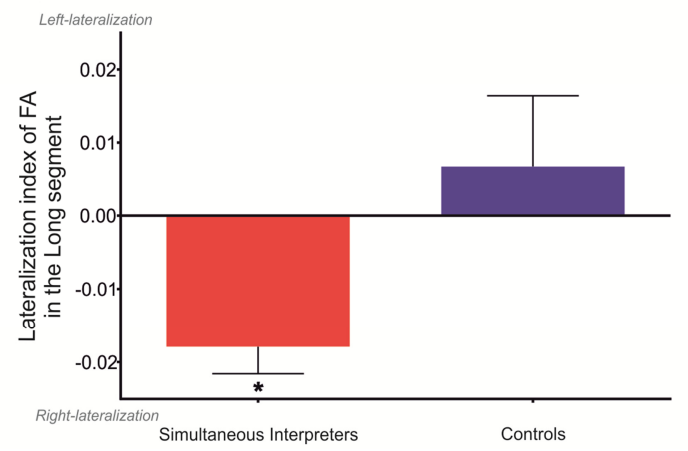

B)

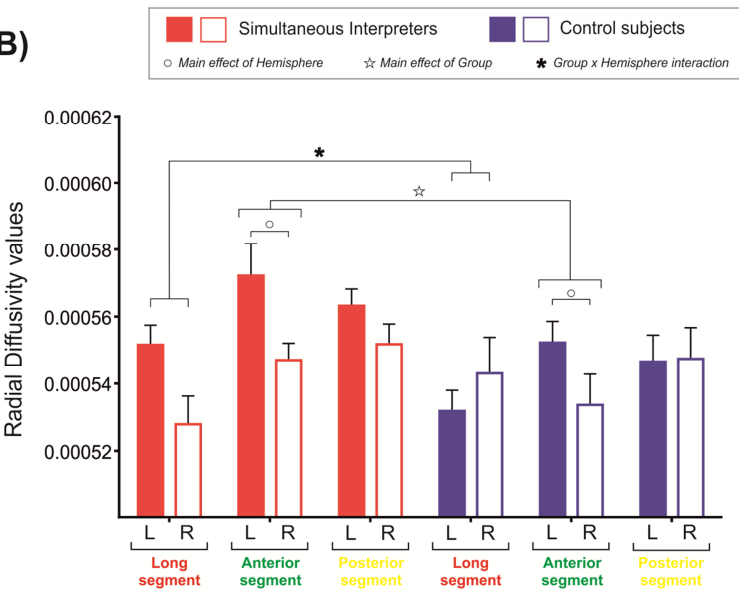

D)

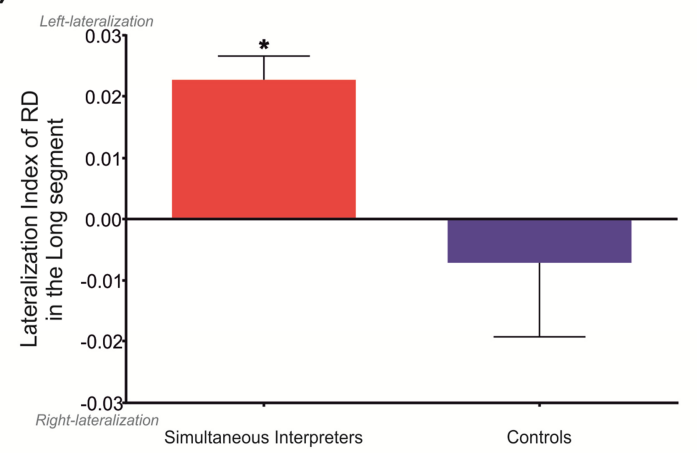

Figure 4. Significant results of the manual deterministic dissection procedure: (A) FA, "group x hemisphere" interaction in the long AF segment and main effect of "group" in the anterior AF segment; (B) RD, "group $\mathrm{x}$ hemisphere" interaction in the long AF segment and main effect of "group" in the anterior AF segment; (C) FA in the long AF segment, LI; (D) RD in the long AF segment, LI. Red = SIs, blue = controls, colored bars $=\mathrm{LH}$, bordered bars $=\mathrm{RH}$.

- Please insert Figure 4 at about here -

\subsection{Probabilistic tractography: brain-training relationships}

In order to further inspect whether the between-group differences we revealed in the LH (i.e., "group $x$ hemisphere" interaction in FA and RD) as well as in the bilateral long and anterior AF segments (i.e., "group $x$ tract" interaction in FA) 
were related to the number of years of interpreting experience, we performed correlative analyses (i.e., Pearson's $r$, two-tailed). The six correlations calculated with FA values and the four ones computed with RD values were Bonferronicorrected (separately for FA and RD) by taking into account the mean correlation between the variables of interest (see methods, corrected $p$ value for $F A=.021$, RD $=.027)$. The correlation analyses did not reveal significant results for FA (left long AF segment: $r=-.092, p=.753$; left anterior AF segment: $r=-.048, p=.87$; bilateral long AF segment: $r=-.174, p=.552$; bilateral anterior AF segment: $r=$ $.073, p=.804$; left ILF: $r=-.477, p=.085$; left UF: $r=-.482, p=.081$ ) or RD (left long AF segment: $r=.198, p=.497$; left anterior AF segment: $r=.124, p=.672$ left ILF: $r=.544, p=.044$; left UF: $r=.424, p=.131$ ) values.

\subsection{Manual deterministic tractography: brain-training relationships}

To further inspect whether the between-group differences we revealed in the long ("group $x$ hemisphere" interaction in FA and RD) and bilateral anterior AF segments (main effect of "group" in FA and RD) were related to the number of years of interpreting experience, we performed additional correlative analyses (i.e., Pearson's r, two-tailed). The two correlations we computed with FA and RD values were Bonferroni-corrected (separately for FA and RD) by taking into account the mean correlation between the variables of interest (see methods, corrected p value for $\mathrm{FA}=.035, \mathrm{RD}=. \mathbf{0 4 4})$. The correlations computed with FA (bilateral anterior AF segment: $r=.299, p=.402 ;$ right long AF segment: $r=-.432, p=.212$ ) and $R D$ (bilateral anterior AF segment: $r=-.283, p=.428$; left long AF segment: $r=.259$, $p$ $=.37)$ did not reach significance. 


\subsection{Correlations between probabilistic and deterministic tractography data}

As an addendum to the main analyses, we compared probabilistic and deterministic data by using a correlative approach (Figure 5). In particular, we correlated mean FA and $\mathrm{RD}$ values of the entire AF, long AF segment, and anterior AF segment within the whole sample of participants, separately for the two hemispheres. These additional statistical analyses revealed overall significant results, however, with the exception of $\mathrm{RD}$ in the entire right AF (Bonferroni-corrected $\mathbf{p}$ value for FA and RD: .05 / 6 = .0083).

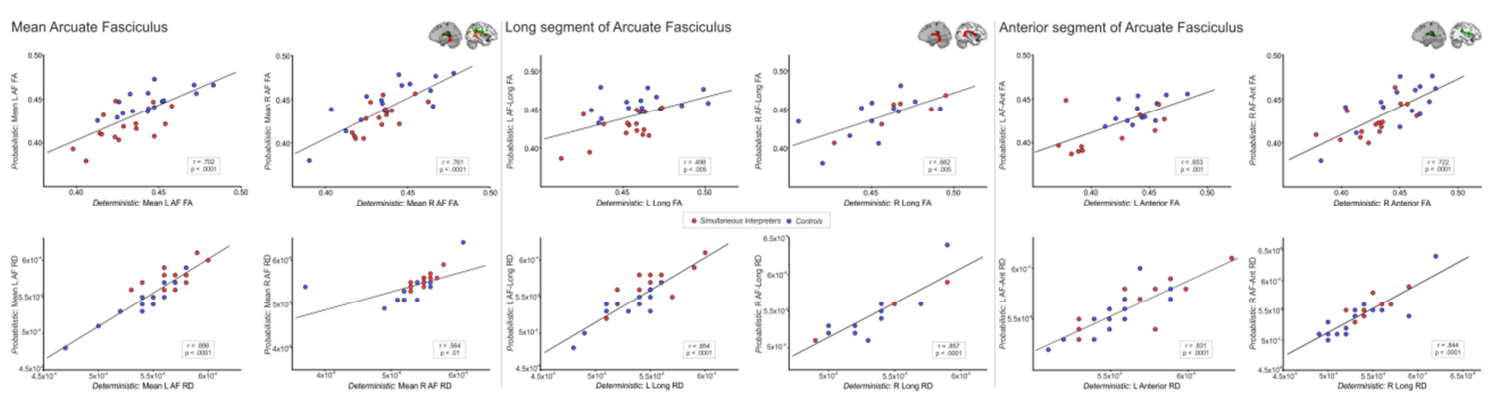

Figure 5. Correlations between probabilistic and deterministic data. First column = entire AF, mean FA (top) and RD (bottom) values in the LH. Second column = entire $\mathrm{AF}$, mean FA (top) and RD (bottom) values in the RH. Third column $=$ long AF segment, mean FA (top) and RD (bottom) values in the LH. Fourth column $=$ long AF segment, mean FA (top) and RD (bottom) values in the RH. Fifth column $=$ anterior AF segment, mean FA (top) and RD (bottom) values in the LH. Sixth column $=$ anterior AF segment, mean FA (top) and RD (bottom) values in the RH. Red = SIs, blue = controls. Abbreviations: L, left hemisphere; R, right hemisphere; AF, Arcuate Fasciculus.

- Please insert Figure 5 about here - 


\section{DISCUSSION}

In the present work, we compared the microstructural properties of the bilateral AF, ILF, and UF between professional SIs and multilingual control participants using probabilistic tractography (Catani et al., 2007; Catani et al., 2002; Catani et al., 2003; Catani et al., 2005; Friederici, 2009; Saur et al., 2008). Furthermore, we re-evaluated the three segments of the AF by means of a deterministic manual dissection procedure. Probabilistic tractography uncovered microstructural between-group differences in the examined pathways of the LH as well as in the bilateral AF. Deterministic tractography revealed between-group differences in the bilateral anterior portion of the AF that were accompanied by expertise-related anatomical peculiarities in the long AF segment. Results are discussed in terms of environmental influences, metaplasticity, and genetic predisposition.

\subsection{Ventral and dorsal processing streams}

The AF has been described as the main pathway supporting the dorsal stream for language processing (Catani et al., 2007; Catani et al., 2005). The left long AF segment connects brain regions involved in "hearing" and "speaking" and is thought to contribute to articulation, auditory-motor integration, and speech imitation (Elmer and Kuhnis, 2016; Lopez-Barroso et al., 2013; Saur et al., 2008; Vaquero et al., 2017). Furthermore, both the left long and anterior AF segments play a crucial role in sentence processing, higher-order linguistic processes as well as verbal working memory (Meyer et al., 2014). Thereby, it is noteworthy to mention that even though an exact functional assignment of the three AF branches is far from unequivocal, the right $\mathrm{AF}$ has been suggested to provide a motor interface to prosody perception (Fruhholz et al., 2015; 
Sammler et al., 2015) and to contribute to verbal memory functions (Catani et al., 2007).

The ventral stream for language functions is supported by the ILF and UF (Catani et al., 2003; Friederici, 2009; Saur et al., 2008). The ILF links ventral-posterior with anterior temporal areas along a hierarchically-organized temporal lobe system and contributes to accessing lexical-semantic information at the word level (Hickok and Poeppel, 2007), processing propositional aspects of speech (Friederici, 2009; Hickok and Poeppel, 2007; Saur et al., 2008), and mapping novel words onto meaning (Ripolles et al., 2017). The UF has been associated with comparable linguistic functions as the ILF (Dick and Tremblay, 2012) but with important computational differences. Through its reciprocal connections with the ventral part of the prefrontal cortex and the temporal pole, the UF mediates top-down influences from the prefrontal cortex to anterior-temporal semantic nodes (Harvey et al., 2013) and facilitates auditory working memory (Dick and Tremblay, 2012), cross-situational word-to-meaning mapping learning (Ripolles et al., 2017), as well as word comprehension in aphasic patients (Harvey et al., 2013).

Based on the functional-anatomical framework described above, the pathway-unspecific between-group differences we revealed in the language-dominant LH might possibly reflect an optimization of neurocognitive aspects of language processing, including lexical-semantic access (Elmer et al., 2010b), articulation, and sensory-to-motor transformations (Elmer et al., 2010a). Otherwise, the microstructural differences we identified in the bilateral and right-sided AF might possibly facilitate working memory functions and the processing of prosodic speech information (Meyer et al., 2014; Sammler et al., 2015). However, in order to provide a clearer picture of the specific 
functions supported by the different fiber tracts in SIs, future studies should integrate functional and anatomical data and screen a variety of language and cognitive functions, including verbal fluency, verbal memory, language switching abilities and executive functions, only to mention a few of them.

\subsection{The pathways to language in simultaneous interpreters}

Currently, only two studies have addressed the relationships between simultaneous interpreting and white matter architecture (Elmer et al., 2010a; Van de Putte et al., 2018). In the first DTI study, Elmer and colleagues used tract-based spatial statistics and performed whole-brain voxel-by-voxel white matter comparisons between SIs and multilingual control participants. As main results, this study consistently revealed lower FA values in SIs in brain regions supporting cognitive control mechanisms, articulation, and sensory-to-motor coupling mechanisms, including the forceps minor, forceps major, left anterior insula, IPL, middle-anterior cingulum, and head of the caudate nucleus (Elmer et al., 2010a). In a second study, Van de Putte and co-workers used networkbased statistics and compared structural connectivity (i.e., number of streamlines) between SIs and translators after nine months of training. The authors reported increased training-related connectivity in SIs in frontal-basal ganglia circuits and cerebellar-supplementary motor area networks possibly contributing to cognitive control during language processing (Van de Putte et al., 2018).

In the present work, we attempted to bridge a gap in the literature, and focused on the microstructural properties of the main fiber tracts of the ventral and dorsal processing streams (Friederici, 2009; Hickok and Poeppel, 2007; Rauschecker and Scott, 2009; Saur et al., 2008). In line with our hypothesis, probabilistic tractography generally 
revealed pathway-unspecific group differences in the language-dominant LH as well as specific effects in the AF. In particular, SIs demonstrated lower FA and higher RD in the LH that was accompanied by reduced FA in the bilateral AF (long and anterior segments). However, importantly, a more meticulous manual dissection of the three segments of the AF showed that the group difference in the bilateral AF was limited to the anterior segment, and reflected by reduced FA and increased RD in SIs compared to controls. Notably, manual AF reconstruction also revealed tract-specific group differences that could not be detected by automated probabilistic tractography, namely increased mean FA in the right and $\mathrm{RD}$ in the left long AF segment in SIs compared to control participants. Taken together, these results indicate a composite of systemic white matter peculiarities in the LH and specific microstructural differences in the long and anterior AF segments.

The systemic reorganization we revealed in SIs in the LH is reconcilable with previous functional neuroimaging studies showing a main contribution of left-hemispheric perisylvian areas to simultaneous language translation (Elmer, 2016; Hervais-Adelman et al., 2015; Rinne et al., 2000). However, since mean FA in the left AF, ILF, and UF was not predictive of the number of years of interpreting experience, our results suggest that the functional implication of the $\mathrm{LH}$ in simultaneous language translation is possibly optimized by pre-existing microstructural characteristics in the underlying white matter architecture. Otherwise, it is still unclear whether the anatomical peculiarities of these tracts might facilitate the high demands placed on language processing per se or rather metaplasticity processes that possibly facilitated "learning to learn" to become a professional SI (Vaquero et al., 2016). Importantly, both probabilistic and deterministic approaches also revealed 
pathway-specific group differences in the long and anterior AF segments. This result is not at all surprising, since simultaneous interpreting strongly relies on auditory-motor integration, working memory, and higher-order linguistic functions (Elmer and Kuhnis, 2016; Morales et al., 2015; Stavrakaki et al., 2012). Since the microstructural properties of the long and anterior AF segments did not correlate with the years of interpreting experience, results are interpreted as indicating an influence of predisposition rather than training-related changes.

A previous source-based EEG study provided first evidence for increased trainingrelated functional connectivity between pST regions and Broca's area in SIs compared to controls during a cross-linguistic semantic decision task (Elmer and Kuhnis, 2016). Increased functional and structural connectivity between the left auditory cortex and Broca's area (long AF segment) has previously also been shown to promote speech segmentation and word learning (Lopez-Barroso et al., 2013). Moreover, Catani and colleagues revealed that a bilateral manifestation of the long AF segment facilitates verbal recall (Catani et al., 2007), whereas the right long AF segment has been associated with prosodic processes and spoken sentence comprehension (Kotz et al., 2003; Sammler et al., 2015). In this vein, it is noteworthy to mention that IP and ventral frontal areas also strongly contribute to verbal working memory functions that are mediated by functional and structural connectivity between frontal and parietal areas (Albouy et al., 2017; Borst and Anderson, 2013; Darki and Klingberg, 2015; Papagno et al., 2017; Schomers et al., 2017).

Interestingly, previous literature on language learning provided evidence showing that a nine month intensive language course leads to white matter reorganizations in language- 
related pathways of the $\mathrm{LH}$, its right-sided homologues as well as in frontal lobe tracts implicated in the coordination of higher cognitive functions (Schlegel et al., 2012). Language-related white matter pathways in perisylvian brain regions have also been proposed to constitute the neural underpinnings of language aptitude variations that are influenced by both linguistic and cognitive functions (Xiang et al., 2012). In particular, Xiang and colleagues found that grammatical inferencing was related to the degree of structural connectivity between posterior-temporal and ventral-frontal areas, whereas vocabulary learning and verbal working memory functions were dependent upon frontoparietal projections.

The results of our study are somewhat in contradiction with those mentioned above in that we mainly revealed decreased FA and increased RD values in SIs compared to controls. However, by taking into account the literature focusing on training in different domains as well as language learning, it becomes evident that there is no clear relationship between diffusion parameters and expertise. For example, professional musicians have previously shown to be characterized by significantly reduced FA values in the corticospinal tract (Imfeld et al., 2009) as well as in the corona radiata and the bilateral internal capsule compared to non-musicians (Schmithorst and Wilke, 2002). Also previous work on bilinguals brought to light language-related white matter constellations that differed from those of monolingual speakers. Recently, Singh et and colleagues (Singh, 2017) evaluated several microstructural white matter parameters between bilinguals and monolinguals and reported a multifaceted pattern of betweengroup differences in the right SLF, right ILF, bilateral SLF and inferior fronto-occipital fasciculus (IFOF). Thereby, the authors identified overall increased FA values in bilinguals compared to monolinguals. However, bilinguals were also characterized by 
higher mean RD values in the right SLF and ILF, and second language (L2) proficiency score was positively related to mean RD in the right SLF. In a further DTI study, Mohades and colleagues (Mohades, 2015) performed repeated DTI measurements with an inter-scan interval of two years in simultaneous-bilinguals, sequential-bilinguals, and monolinguals and did not reveal significant FA changes in the left AF and SLF. However, sequential-bilinguals demonstrated the most prominent FA changes in the left IFOF, and FA in the same tract was higher in simultaneous-bilinguals compared to monolinguals. Last, but not least, Vandermosten and colleagues (Vandermosten et al., 2015) reported reduced FA values in the left long AF segment in phoneticians compared to control participants that negatively correlated with the number of years of phonetic training. Taken together, previous studies focusing on expertise in different domains reported controversial findings that render the interpretation of different diffusion parameters difficult, possibly because FA and RD values also vary as a function of biographical characteristics (Hamalainen et al., 2017). In the next section, we will provide more differentiated arguments that may explain the between-group differences we revealed in terms of FA and RD values.

Finally, it should be mentioned that cross-sectional approaches have the drawback that they do not enable to build causal relationships between white matter changes and linguistic or cognitive functions. In fact, such clear relationships can only be addressed using longitudinal designs and by combining multimodal imaging parameters with a holistic psychological and linguistic assessment. Accordingly, we cannot exclude that the white matter differences we revealed between SIs and controls might be related to other factors than language expertise per se. Furthermore, even though the control participants were highly fluent multilingual speakers, we cannot exclude that the two 
groups differed in some aspects of language proficiency, including grammar skills, vocabulary, or verbal fluency. In a similar vein, it might be possible that the two groups differed in their daily usage of languages or in terms of cognitive functions. In the present work, we explicitly measured participants with a university background. However, we cannot rule out that the two groups differed in some cognitive dimensions that were potentially influenced by multilingual speech competence, although such an influence is controversial (Paap et al., 2016; van der Linden et al., 2018).

\subsection{Interpretation of $F A$ and $R D$ values}

Myelin integrity has commonly been associated with increased FA and reduced RD values (Beaulieu, 2002; Mori and Zhang, 2006). However, this dichotomy is too simplistic and does not explain previous DTI findings showing a reversed diffusivity pattern to that classically envisaged in several groups of participants undergoing longterm training (Bengtsson et al., 2005; Elmer et al., 2010a; Imfeld et al., 2009; Vandermosten et al., 2015). Nowadays, there is a common belief that re-myelination in the adult brain generally fails (Filbin, 2003). Accordingly, it is conceivable to assume that white matter plasticity in adulthood involves mechanisms other than re-myelination per se. One possibility accounting for the overall lower FA and higher RD values we revealed in SIs is that intensive language training in adulthood leads to increased axon diameters, resulting in an expansion of the extracellular space and hence in higher action potential conduction velocity due to less resistance from the cell membrane (Beaulieu, 2002). Another possibility is that lower FA and higher RD in SIs reflect an increased fiber complexity or crossing fibers (Wedeen et al., 2008). A further interpretation is that SIs are more susceptible to training-related axonal pruning that may result in a relative change of the hemispheric balance of myelin integrity (Elmer et 
al., 2014). Such a change in asymmetric weighting of microstructural white matter properties is in line with the LI results, indicating generally higher FA and lower RD values in the RH compared to the LH in SIs. Finally, there is also the possibility that the between-group differences we revealed in the LH might be related to microstructural peculiarities in fiber tracts that have not been evaluated in the present study. In this context, we cannot exclude that reduced FA and increased RD values in the LH of SIs might reflect fiber complexity that originated from an increased number of orthogonal or crossing fibers.

\subsection{Compliance between probabilistic and deterministic tractography data}

In the present study, we also compared bilateral mean FA and RD values of the entire AF, long AF segment, and anterior AF segment between the two methods. Overall, the correlative analyses pointed to medium to high consistencies between the two procedures, with an explained variance in the range of $24.8-80.6 \%$. However, surprisingly, the consistency was higher for RD than FA values, leading to suggest that FA is more strongly influenced by the dissection procedure used. Even though these analyses did not constitute the main part of our work, our results could be useful for better comprehending divergent tractography findings. Certainly, future methodological studies should more carefully evaluate the utility and robustness of the two dissection procedures in order to meticulously identify the circumstances in which one specific method is more efficient and appropriate than the other one.

\section{LIMITATIONS}

In the present work, we have to acknowledge some limitations. A first shortcoming of this study is that we did not compare cognitive abilities and executive functions between 
the two groups, rendering it difficult to relate white matter differences to specific psychological functions. Even though it is not yet clear whether interpreting training might promote cognitive functions more strongly than multilingualism per se (Aparicio et al., 2017; Henrard and Van Daele, 2017; van der Linden et al., 2018), we cannot exclude that some between-group differences in cognitive functioning might have influenced the results. A second shortcoming of our work is that we did not explicitly test the language competence of the participants but only required that they were able to fluently speak the foreign languages in a conversational context (i.e., based on selfreport). We also did not measure language proficiency because it would have been too demanding to test the proficiency of all spoken languages (i.e., the participants spoke 7 different languages) using the same test procedure. Furthermore, we cannot exclude that control participants and SIs somehow differed in terms of language usage. In particular, it is possible that due to living and working in a multilingual environment, in every day's life the control participants switched more frequently between languages compared to SIs. Finally, it should be mentioned that the participants we measured in this study were characterized by a certain inter-individual variability in terms of languages spoken. Accordingly, we cannot exclude that this variable or the frequency of usage of different languages might have influenced our results.

\section{CONCLUSIONS}

In the present work, we compared a specific set of white matter pathways that have previously been identified as essential for speech processing (Friederici, 2009), namely the AF, ILF, and UF. Results revealed a composite of pathwayspecific and unspecific between-group differences. In particular, we consistently revealed reduced mean $F A$ and increased mean $R D$ in SIs compared to 
multilingual control participants in the analyzed pathways of the LH that resulted in an asymmetric weighting of microstructural white matter properties. Furthermore, we detected bilateral between-group differences in the long and anterior AF segments. Since microstructural parameters did not correlate with the number of years of interpreting experience, results are interpreted as reflecting predisposition rather than training-related plasticity. These results have important implications for better comprehending the complex relationships between white matter organization and language expertise in adulthood.

\section{ACKNOWLEDMENTS}

This research was supported by the Swiss National Science Foundation (SNF, grant nr. 320030_163149 to Lutz Jäncke). CF was supported by a Spanish MINECO project (PSI2015-69132P) and by the Catalan Government (Generalitat de Catalunya, PERIS2017). LV was partially supported by the Quebec Bio-Imaging Network (QBIN) and the Fonds de Recherche du Québec-Nature et Technologies (Merit Scholarship Program for Foreign Students). We would like to thank Lutz Jäncke for having provided the necessary infrastructure and technology for the study as well as for his financial support. No conflicts of interest are declared.

\section{AUTHOR CONTRIBUTIONS}

SE, JH, and ARF planned the study, SE performed the MRI measurements, and JH, LV, and GOC evaluated the DTI data. SE, LV, and GOC performed the statistical analyses, and drafted the manuscript together with JH, CF, and ARF. SE, JH, LV, GOC, CF, and ARF contributed to the interpretation of the data. GOV manually dissected the three AF 
segments. LV supervised and reviewed the manual dissections and arranged all the figures.

\section{TABLES LEGEND}

Table 1. Biographical data of the participants. GER = German; FR = French; ENG $=$ English; IT = Italian; $\mathrm{SP}=$ Spanish; $\mathrm{POR}=$ Portuguese DU $=$ Dutch. $\mathrm{SI}=$ simultaneous interpreters; $\mathrm{CON}=$ multilingual control participants.

\section{FIGURES LEGEND}

Figure 1. Automated probabilistic tractography reconstructions of the four languagerelated tracts in three simultaneous interpreters (upper row) and three control participants (lower row). Green $=$ anterior segment of the Arcuate Fasciculus, red $=$ long segment of the Arcuate Fasciculus, blue $=$ Inferior Longitudinal Fasciculus, magenta $=$ Uncinate Fasciculus Abbreviations: Ant, anterior; Fasc., fasciculus; Inf., inferior; L, left; $x, y, z$, Montreal Neurological Institute space coordinates of the background slices.

Figure 2. Manual deterministic tractography reconstructions of the three segments of the arcuate fasciculus (AF) in three simultaneous interpreters (upper row) and three control participants (lower row). Green $=$ anterior segment of the Arcuate Fasciculus, red $=$ long segment of the Arcuate Fasciculus, yellow $=$ posterior segment of the Arcuate Fasciculus. Abbreviations: $\mathrm{L}=1 \mathrm{eft} ; \mathrm{x}, \mathrm{y}, \mathrm{z}=$ Montreal Neurological Institute space coordinates of the background slices. 
Figure 3. Significant results of the automated probabilistic tractography procedure: (A) FA, "group $\mathrm{x}$ hemisphere" and "group $\mathrm{x}$ tract" interactions; (B) RD, "group $\mathrm{x}$ hemisphere" interaction; (C) FA, "group x hemisphere" interaction; (D) RD, "group x hemisphere" interaction; (E) mean FA of all tracts, LI; (F) mean RD of all tracts, LI. Red $=$ SIs, blue $=$ controls, colored bars $=\mathrm{LH}$, bordered bars $=$ RH. Abbreviations: AFLong, long segment of the Arcuate Fasciculus; AF-Ant, anterior segment of the Arcuate Fasciculus; ILF, inferior Longitudinal Fasciculus; UF, Uncinate Fasciculus.

Figure 4. Significant results of the manual deterministic dissection procedure: (A) FA, "group x hemisphere" interaction in the long AF segment and main effect of "group" in the anterior AF segment; (B) RD, "group $\mathrm{x}$ hemisphere" interaction in the long $\mathrm{AF}$ segment and main effect of "group" in the anterior AF segment; (C) FA in the long AF segment, LI; (D) RD in the long AF segment, LI. Red = SIs, blue = controls, colored bars $=\mathrm{LH}$, bordered bars $=\mathrm{RH}$.

Figure 5. Correlations between probabilistic and deterministic data. First column $=$ entire AF, mean FA (top) and RD (bottom) values in the LH. Second column = entire AF, mean FA (top) and RD (bottom) values in the RH. Third column = long AF segment, mean FA (top) and RD (bottom) values in the LH. Fourth column = long AF segment, mean FA (top) and RD (bottom) values in the RH. Fifth column = anterior AF segment, mean FA (top) and RD (bottom) values in the LH. Sixth column = anterior AF segment, mean FA (top) and RD (bottom) values in the RH. Red = SIs, blue $=$ controls. Abbreviations: L, left hemisphere; R, right hemisphere; AF, Arcuate Fasciculus. 


\section{REFERENCES}

Albouy, P., Weiss, A., Baillet, S., Zatorre, R.J., 2017. Selective Entrainment of Theta Oscillations in the Dorsal Stream Causally Enhances Auditory Working Memory Performance. Neuron 94, 193-206 e195.

Annett, M., 1970. A Classification of Hand Preference by Association Analysis. British Journal of Psychology 61, 303-\&.

Aparicio, X., Heidlmayr, K., Isel, F., 2017. Inhibition Efficiency in Highly Proficient Bilinguals and Simultaneous Interpreters: Evidence from Language Switching and Stroop Tasks. Journal of Psycholinguistic Research 46, 1427-1451.

Beaulieu, C., 2002. The basis of anisotropic water diffusion in the nervous system - a technical review. NMR Biomed 15, 435-455.

Becker, M., Schubert, T., Strobach, T., Gallinat, J., Kuhn, S., 2016. Simultaneous interpreters vs. professional multilingual controls: Group differences in cognitive control as well as brain structure and function. Neuroimage 134, 250-260.

Bengtsson, S.L., Nagy, Z., Skare, S., Forsman, L., Forssberg, H., Ullen, F., 2005. Extensive piano practicing has regionally specific effects on white matter development. Nat Neurosci 8, 1148-1150.

Bezzola, L., Merillat, S., Gaser, C., Jancke, L., 2011. Training-induced neural plasticity in golf novices. J Neurosci 31, 12444-12448.

Borst, J.P., Anderson, J.R., 2013. Using model-based functional MRI to locate working memory updates and declarative memory retrievals in the fronto-parietal network. Proceedings of the National Academy of Sciences of the United States of America 110, 1628-1633.

Catani, M., Allin, M.P., Husain, M., Pugliese, L., Mesulam, M.M., Murray, R.M., Jones, D.K., 2007. Symmetries in human brain language pathways correlate with verbal recall. Proc Natl Acad Sci U S A 104, 17163-17168.

Catani, M., Howard, R.J., Pajevic, S., Jones, D.K., 2002. Virtual in vivo interactive dissection of white matter fasciculi in the human brain. Neuroimage 17, 77-94.

Catani, M., Jones, D.K., Donato, R., Ffytche, D.H., 2003. Occipito-temporal connections in the human brain. Brain 126, 2093-2107.

Catani, M., Jones, D.K., ffytche, D.H., 2005. Perisylvian language networks of the human brain. Ann Neurol 57, 8-16.

Catani, M., Mesulam, M., 2008. The arcuate fasciculus and the disconnection theme in language and aphasia: History and current state. Cortex 44, 953-961.

Corballis, M.C., 2010. Mirror neurons and the evolution of language. Brain and Language 112, 25-35.

Darki, F., Klingberg, T., 2015. The Role of Fronto-Parietal and Fronto-Striatal Networks in the Development of Working Memory: A Longitudinal Study. Cerebral Cortex 25, 1587-1595.

Dick, A.S., Tremblay, P., 2012. Beyond the arcuate fasciculus: consensus and controversy in the connectional anatomy of language. Brain 135, 3529-3550.

Elmer, S., 2016. Broca Pars Triangularis Constitutes a "Hub" of the Language-Control Network during Simultaneous Language Translation. Front Hum Neurosci 10, 491.

Elmer, S., Hanggi, J., Jancke, L., 2014. Processing demands upon cognitive, linguistic, and articulatory functions promote grey matter plasticity in the adult multilingual brain: Insights from simultaneous interpreters. Cortex 54, 179-189. 
Elmer, S., Hänggi, J., Meyer, M., Jäncke, L., 2010a. Differential language expertise related to white matter architecture in regions subserving sensory-motor coupling, articulation, and interhemispheric transfer. Human Brain Mapping [Epub ahead of print], 12.

Elmer, S., Kuhnis, J., 2016. Functional Connectivity in the Left Dorsal Stream Facilitates Simultaneous Language Translation: An EEG Study. Front Hum Neurosci 10,60 .

Elmer, S., Meyer, M., Jancke, L., 2010b. Simultaneous interpreters as a model for neuronal adaptation in the domain of language processing. Brain Res 1317, 147-156.

Filbin, M.T., 2003. Myelin-associated inhibitors of axonal regeneration in the adult mammalian CNS. Nat Rev Neurosci 4, 703-713.

Friederici, A.D., 2009. Pathways to language: fiber tracts in the human brain. Trends in Cognitive Sciences 13, 175-181.

Friederici, A.D., 2012. The cortical language circuit: from auditory perception to sentence comprehension. Trends in Cognitive Sciences 16, 262-268.

Fruhholz, S., Gschwind, M., Grandjean, D., 2015. Bilateral dorsal and ventral fiber pathways for the processing of affective prosody identified by probabilistic fiber tracking. Neuroimage 109, 27-34.

Hamalainen, S., Sairanen, V., Leminen, A., Lehtonen, M., 2017. Bilingualism modulates the white matter structure of language-related pathways. Neuroimage 152, 249-257.

Harvey, D.Y., Wei, T., Ellmore, T.M., Hamilton, A.C., Schnur, T.T., 2013. Neuropsychological evidence for the functional role of the uncinate fasciculus in semantic control. Neuropsychologia 51, 789-801.

Havas, V., Laine, M., Rodriguez Fornells, A., 2017. Brain signatures of early lexical and morphological learning of a new language. Neuropsychologia 101, 47-56.

Henrard, S., Van Daele, A., 2017. Different Bilingual Experiences Might Modulate Executive Tasks Advantages: Comparative Analysis between Monolinguals, Translators, and Interpreters. Frontiers in Psychology 8.

Hervais-Adelman, A., Moser-Mercer, B., Golestani, N., 2015. Brain functional plasticity associated with the emergence of expertise in extreme language control. Neuroimage 114, 264-274.

Hervais-Adelman, A., Moser-Mercer, B., Murray, M.M., Golestani, N., 2017. Cortical thickness increases after simultaneous interpretation training. Neuropsychologia 98, 212-219.

Hickok, G., Poeppel, D., 2007. Opinion - The cortical organization of speech processing. Nature Reviews Neuroscience 8, 393-402.

Hyde, K.L., Lerch, J., Norton, A., Forgeard, M., Winner, E., Evans, A.C., Schlaug, G., 2009. Musical Training Shapes Structural Brain Development. Journal of Neuroscience 29, 3019-3025.

Imfeld, A., Oechslin, M.S., Meyer, M., Loenneker, T., Jancke, L., 2009. White matter plasticity in the corticospinal tract of musicians: a diffusion tensor imaging study. Neuroimage 46, 600-607.

Joanisse, M.F., Zevin, J.D., McCandliss, B.D., 2007. Brain mechanisms implicated in the preattentive categorization of speech sounds revealed using fMRI and a shortinterval habituation trial paradigm. Cerebral Cortex 17, 2084-2093.

Klein, D., Zatorre, R.J., Chen, J.K., Milner, B., Crane, J., Belin, P., Bouffard, M., 2006. Bilingual brain organization: a functional magnetic resonance adaptation study. Neuroimage 31, 366-375. 
Kotz, S.A., Meyer, M., Alter, K., Besson, M., von Cramon, D.Y., Friederici, A.D., 2003. On the lateralization of emotional prosody: an event-related functional MR investigation. Brain Lang 86, 366-376.

Kroll, J.F., de Groot, A.M.B., 2005. Simultaneous interpreting: a cognitive perspective. Handbook of bilingualism. Oxford University Press, pp. 454-479.

Liberman, A.M., Mattingly, I.G., 1985. The Motor Theory of Speech-Perception Revised. Cognition 21, 1-36.

Lopez-Barroso, D., Catani, M., Ripolles, P., Dell'Acqua, F., Rodriguez-Fornells, A., Diego-Balaguer, R., 2013. Word learning is mediated by the left arcuate fasciculus. Proceedings of the National Academy of Sciences of the United States of America 110, 13168-13173.

Meyer, L., Cunitz, K., Obleser, J., Friederici, A.D., 2014. Sentence processing and verbal working memory in a white-matter-disconnection patient. Neuropsychologia 61, 190-196.

Mohades, S.G.V.S., P.; Rosseel, Y.; Van De Craen, P.; Luypaert, R.; Baeken, C., 2015. White-matter development is different in bilingual and monolingual children: a longitudinal DTI study. Plos One 10.

Morales, J., Padilla, F., Gomez-Ariza, C.J., Bajo, M.T., 2015. Simultaneous interpretation selectively influences working memory and attentional networks. Acta Psychol (Amst) 155, 82-91.

Mori, S., Zhang, J., 2006. Principles of diffusion tensor imaging and its applications to basic neuroscience research. Neuron 51, 527-539.

Obleser, J., Eisner, F., Kotz, S.A., 2008. Bilateral speech comprehension reflects differential sensitivity to spectral and temporal features. Journal of Neuroscience 28, 8116-8123.

Paap, K.R., Johnson, H.A., Sawi, O., 2016. Should the search for bilingual advantages in executive functioning continue? Cortex 74, 305-314.

Palomar-Garcia, M.A., Sanjuan, A., Bueicheku, E., Ventura-Campos, N., Avila, C., 2017. The dynamic imprint of word learning on the dorsal language pathway. Neuroimage 159, 261-269.

Papagno, C., Comi, A., Riva, M., Bizzi, A., Vernice, M., Casarotti, A., Fava, E., Bello, L., 2017. Mapping the Brain Network of the Phonological Loop. Human Brain Mapping 38, 3011-3024.

Price, C.J., Green, D.W., von Studnitz, R., 1999. A functional imaging study of translation and language switching. Brain 122 ( Pt 12), 2221-2235.

Proverbio, A.M., Leoni, G., Zani, A., 2004. Language switching mechanisms in simultaneous interpreters: an ERP study. Neuropsychologia 42, 1636-1656.

Rauschecker, J.P., Scott, S.K., 2009. Maps and streams in the auditory cortex: nonhuman primates illuminate human speech processing. Nature Neuroscience 12, 718724.

Rilling, J.K., Glasser, M.F., Preuss, T.M., Ma, X.Y., Zhao, T.J., Hu, X.P., Behrens, T.E.J., 2008. The evolution of the arcuate fasciculus revealed with comparative DTI. Nature Neuroscience 11, 426-428.

Rinne, J.O., Tommola, J., Laine, M., Krause, B.J., Schmidt, D., Kaasinen, V., Teras, M., Sipila, H., Sunnari, M., 2000. The translating brain: cerebral activation patterns during simultaneous interpreting. Neurosci Lett 294, 85-88.

Ripolles, P., Biel, D., Penaloza, C., Kaufmann, J., Marco-Pallares, J., Noesselt, T., Rodriguez-Fornells, A., 2017. Strength of Temporal White Matter Pathways Predicts Semantic Learning. J Neurosci. 
Ruff, S., Marie, N., Celsis, P., Cardebat, D., Demonet, J.F., 2003. Neural substrates of impaired categorical perception of phonemes in adult dyslexics: An fMRI study. Brain and Cognition 53, 331-334.

Sammler, D., Grosbras, M.H., Anwander, A., Bestelmeyer, P.E., Belin, P., 2015. Dorsal and Ventral Pathways for Prosody. Curr Biol 25, 3079-3085.

Saur, D., Kreher, B.W., Schnell, S., Kummerer, D., Kellmeyer, P., Vry, M.S., Umarova, R., Musso, M., Glauche, V., Abel, S., Huber, W., Rijntjes, M., Hennig, J., Weiller, C., 2008. Ventral and dorsal pathways for language. Proc Natl Acad Sci U S A 105, 1803518040.

Schlegel, A.A., Rudelson, J.J., Tse, P.U., 2012. White Matter Structure Changes as Adults Learn a Second Language. Journal of Cognitive Neuroscience 24, 1664-1670.

Schmithorst, V.J., Wilke, M., 2002. Differences in white matter architecture between musicians and non-musicians: a diffusion tensor imaging study. Neuroscience Letters 321, 57-60.

Schomers, M.R., Garagnani, M., Pulvermuller, F., 2017. Neurocomputational Consequences of Evolutionary Connectivity Changes in Perisylvian Language Cortex. Journal of Neuroscience 37, 3045-3055.

Singh, N.C.R., A.; Malagi, A.; Ramanujan, K.; Canini, M.; Della Rosa, P.; Raghunathan, P.; Weeks, B.; Abutalebi, J., 2017. Microstructural anatomical differences between bilinguals and monolinguals. Bilingualism: Language and Cognition.

Stavrakaki, S., Megari, K., Kosmidis, M.H., Apostolidou, M., Takou, E., 2012. Working memory and verbal fluency in simultaneous interpreters. Journal of Clinical and Experimental Neuropsychology 34, 624-633.

Turkeltaub, P.E., Coslett, H.B., 2010. Localization of sublexical speech perception components. Brain and Language 114, 1-15.

Uitenbroek, D.G., 1997. SISA Binomial. In: Uitenbroek., S.D.G. (Ed.), Retrieved January 01, 2002, from the World Wide Web: http://home.clara.net/sisa/binomial.htm.

Van de Putte, E., De Baene, W., Garcia-Penton, L., Woumans, E., Dijkgraaf, A., Duyck, W., 2018. Anatomical and functional changes in the brain after simultaneous interpreting training: A longitudinal study. Cortex 99, 243-257.

van der Linden, L., van de Puttee, E., Woumans, E., Duyck, W., Szmalec, A., 2018. Does Extreme Language Control Training Improve Cognitive Control? Comparison of Professional Interpreters, L2 Teachers and Monolinguals. Frontiers in Psychology 9.

Vandermosten, M., Boets, B., Poelmans, H., Sunaert, S., Wouters, J., Ghesquiere, P., 2012. A tractography study in dyslexia: neuroanatomic correlates of orthographic, phonological and speech processing. Brain 135, 935-948.

Vandermosten, M., Price, C.J., Golestani, N., 2015. Plasticity of white matter connectivity in phonetics experts. Brain Structure and Function [Epub ahead of print].

Vaquero, L., Hartmann, K., Ripolles, P., Rojo, N., Sierpowska, J., Francois, C., Camara, E., van Vugt, F.T., Mohammadi, B., Samii, A., Munte, T.F., Rodriguez-Fornells, A., Altenmuller, E., 2016. Structural neuroplasticity in expert pianists depends on the age of musical training onset. Neuroimage 126, 106-119.

Vaquero, L., Ramos-Escobar, N., Francois, C., Penhune, V., Rodriguez-Fornells, A., 2018. White-matter structural connectivity predicts short-term melody and rhythm learning in non-musicians. Neuroimage 181, 252-262.

Vaquero, L., Rodriguez-Fornells, A., Reiterer, S.M., 2017. The Left, The Better: WhiteMatter Brain Integrity Predicts Foreign Language Imitation Ability. Cereb Cortex 27, 3906-3917.

Wedeen, V.J., Wang, R.P., Schmahmann, J.D., Benner, T., Tseng, W.Y., Dai, G., Pandya, D.N., Hagmann, P., D'Arceuil, H., de Crespigny, A.J., 2008. Diffusion 
spectrum magnetic resonance imaging (DSI) tractography of crossing fibers. Neuroimage 41, 1267-1277.

Xiang, H.D., Dediu, D., Roberts, L., van Oort, E., Norris, D.G., Hagoort, P., 2012. The Structural Connectivity Underpinning Language Aptitude, Working Memory, and IQ in the Perisylvian Language Network. Language Learning 62, 110-130.

Yendiki, A., Panneck, P., Srinivasan, P., Stevens, A., Zollei, L., Augustinack, J., Wang, R., Salat, D., Ehrlich, S., Behrens, T., Jbabdi, S., Gollub, R., Fischl, B., 2011. Automated probabilistic reconstruction of white-matter pathways in health and disease using an atlas of the underlying anatomy. Front Neuroinform 5, 23.

Yendiki, A., Reuter, M., Wilkens, P., Rosas, H.D., Fischl, B., 2016. Joint reconstruction of white-matter pathways from longitudinal diffusion MRI data with anatomical priors. Neuroimage 127, 277-286. 


\begin{tabular}{|c|c|c|c|c|c|}
\hline Group & Participant & Age & Gender & Number of experience years & Spoken languages \\
\hline \multirow[t]{15}{*}{ SI } & 1 & 36 & $f$ & 2 & GER, POR, ENG \\
\hline & 2 & 46 & $f$ & 22 & GER, ENG, FR, IT, SP \\
\hline & 3 & 48 & $f$ & 15 & GER, IT, FR \\
\hline & 4 & 32 & $f$ & 4.5 & GER, SP, ENG, FR, POR \\
\hline & 5 & 41 & $f$ & 10 & GER, FR \\
\hline & 6 & 37 & $\mathrm{~m}$ & 11 & GER, EN, FR, SP \\
\hline & 7 & 34 & $\mathrm{~m}$ & 5 & GER, ENG, FR \\
\hline & 8 & 39 & $\mathrm{~m}$ & 8 & GER, ENG, FR \\
\hline & 9 & 36 & $f$ & 9 & GER, ENG \\
\hline & 10 & 35 & $f$ & 9 & GER, FR \\
\hline & 11 & 39 & $f$ & & GER, ENG, FR, SP \\
\hline & 12 & 33 & $f$ & 2 & GER, IT, FR, ENG, SP \\
\hline & 13 & 38 & $f$ & 14 & GER, IT, FR, ENG \\
\hline & 14 & 50 & $f$ & 22 & GER, IT, FR, ENG \\
\hline & 15 & 31 & $\mathrm{~m}$ & 4 & IT; ENG; FR; GER \\
\hline \multirow[t]{16}{*}{$\mathrm{CON}$} & 1 & 26 & $\mathrm{~m}$ & & GER, ENG \\
\hline & 2 & 27 & $f$ & & GER; POR; ENG, DU \\
\hline & 3 & 26 & $f$ & & GER; ENG; FR \\
\hline & 4 & 27 & $f$ & & GER, ENG \\
\hline & 5 & 34 & $\mathrm{~m}$ & & GER, FR, ENG \\
\hline & 6 & 30 & $\mathrm{~m}$ & & SP, GER, ENG \\
\hline & 7 & 28 & $\mathrm{~m}$ & & FR, GER, ENG \\
\hline & 8 & 27 & $f$ & & GER, ENG, FR \\
\hline & 9 & 26 & $f$ & & GER, ENG, FR \\
\hline & 10 & 25 & $f$ & & GER, ENG, FR \\
\hline & 11 & 25 & $f$ & & GER, ENG, FR \\
\hline & 12 & 46 & $f$ & & GER, ENG, FR \\
\hline & 13 & 42 & $f$ & & GER, ENG, FR \\
\hline & 14 & 39 & $f$ & & GER, ENG, FR \\
\hline & 15 & 42 & $f$ & & GER, ENG, FR \\
\hline & 16 & 43 & $\mathrm{~m}$ & & GER, ENG, FR \\
\hline
\end{tabular}


Simultaneous Interpreters
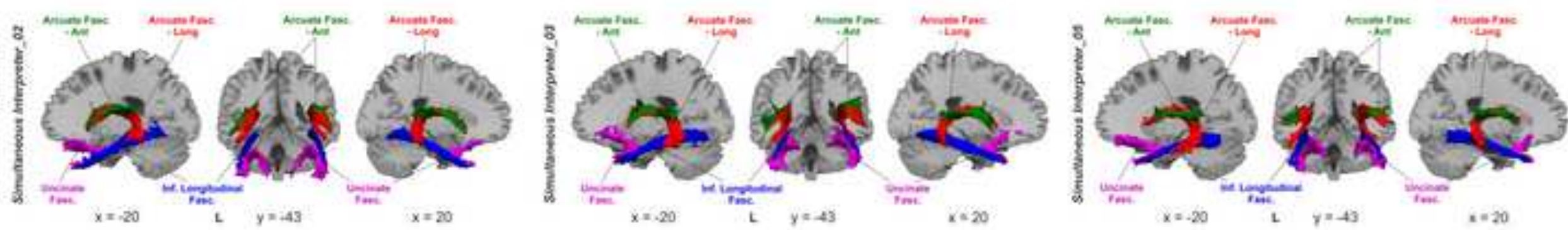

\section{Controls}
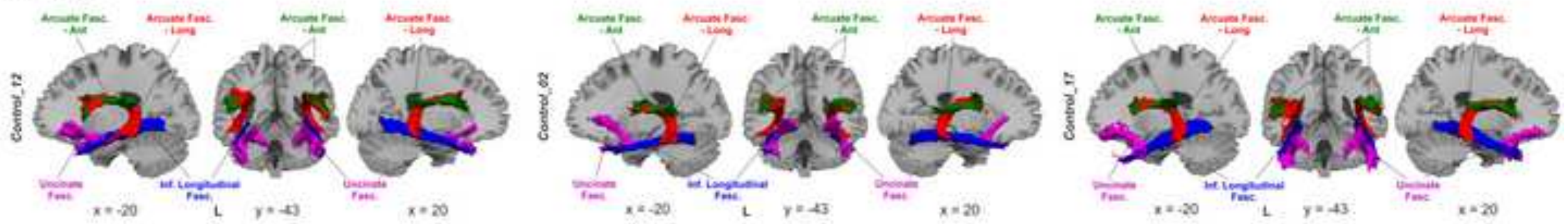
Simultaneous Interpreters
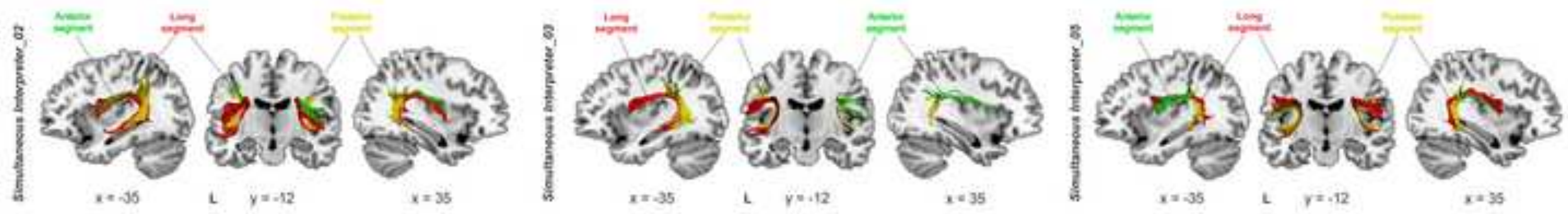

\section{Controls}
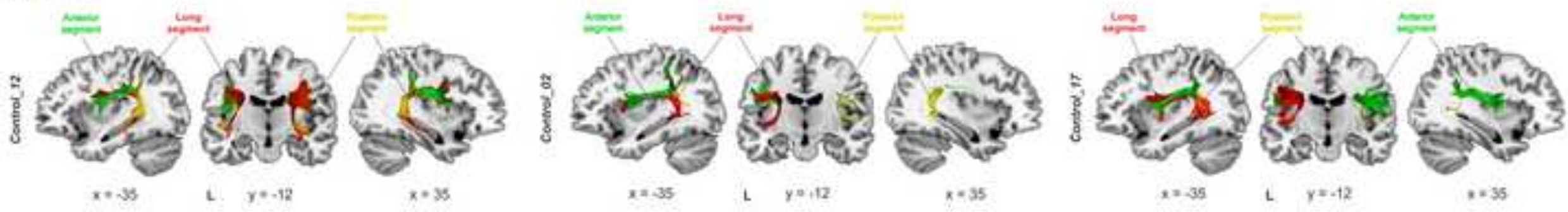

L $\quad y=-12$ 

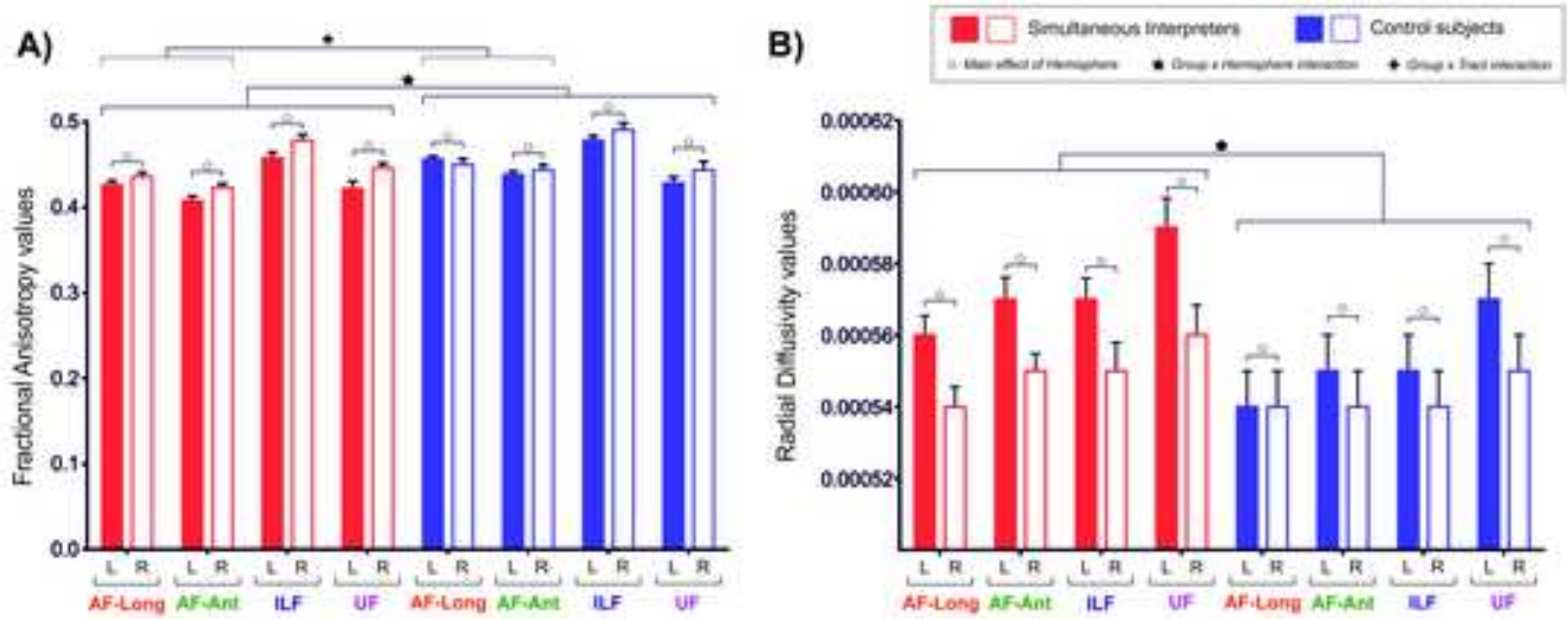

C)

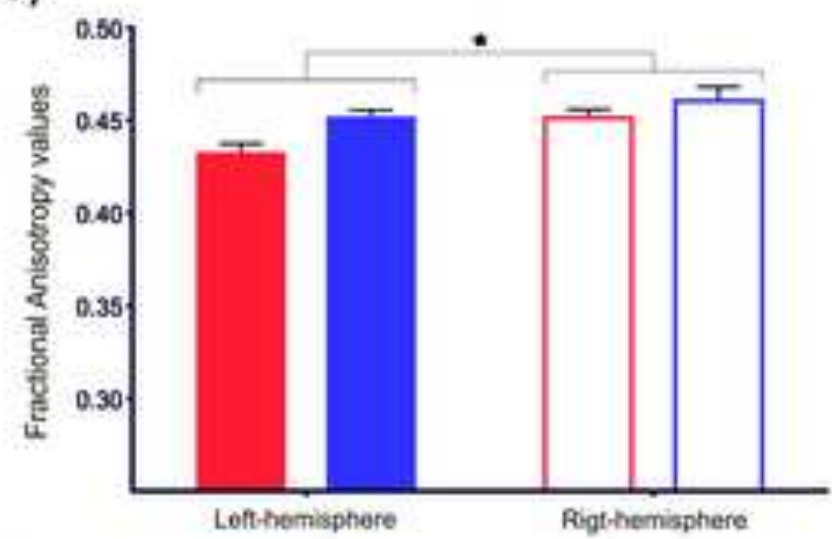

D)
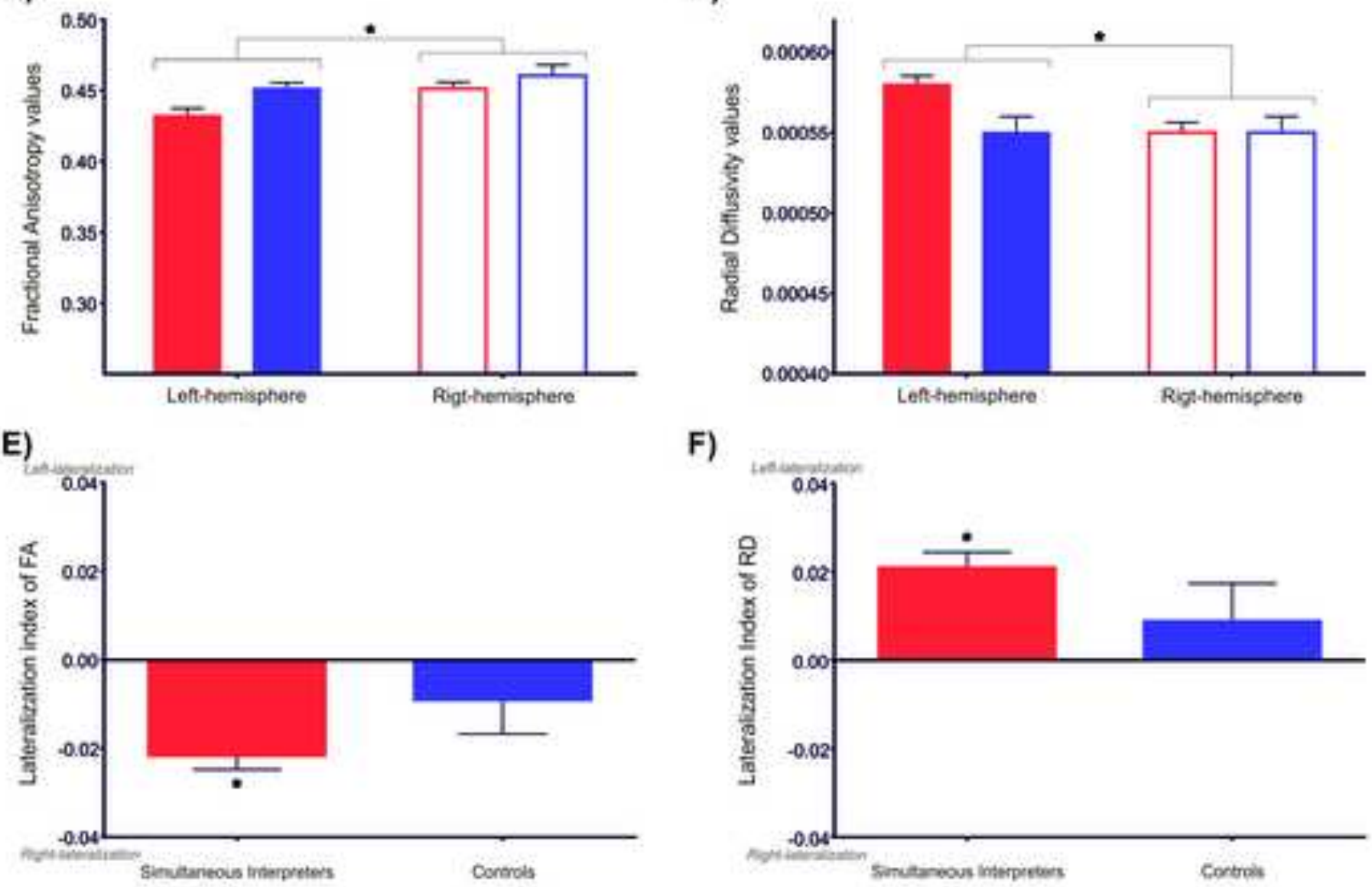

F)

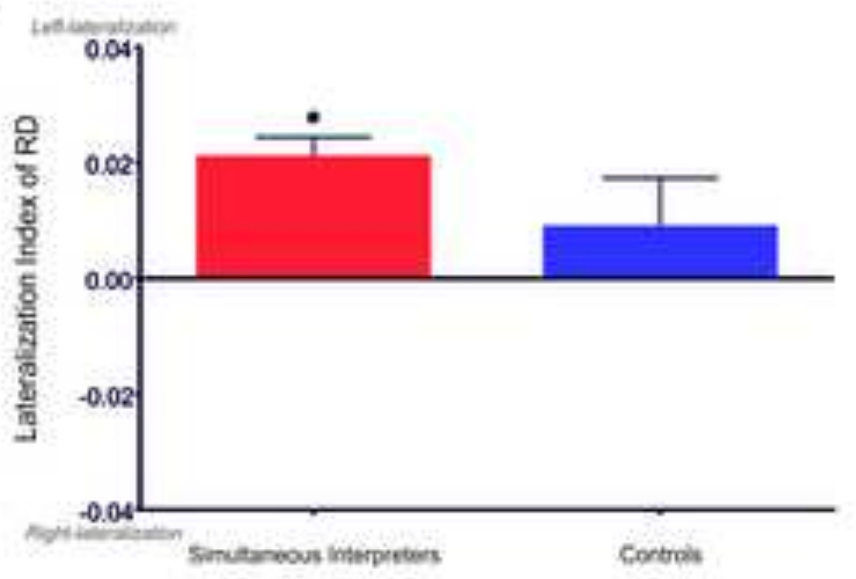


A)

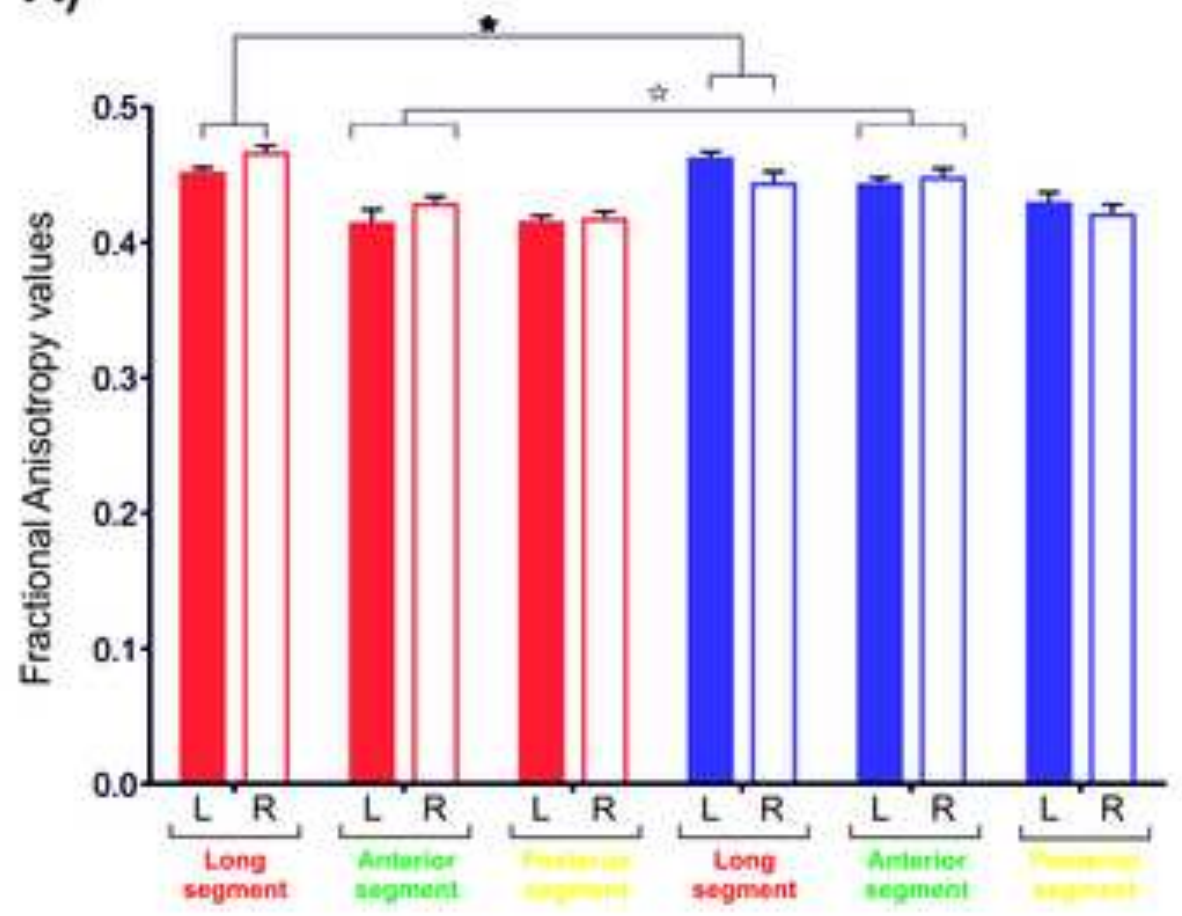

C)

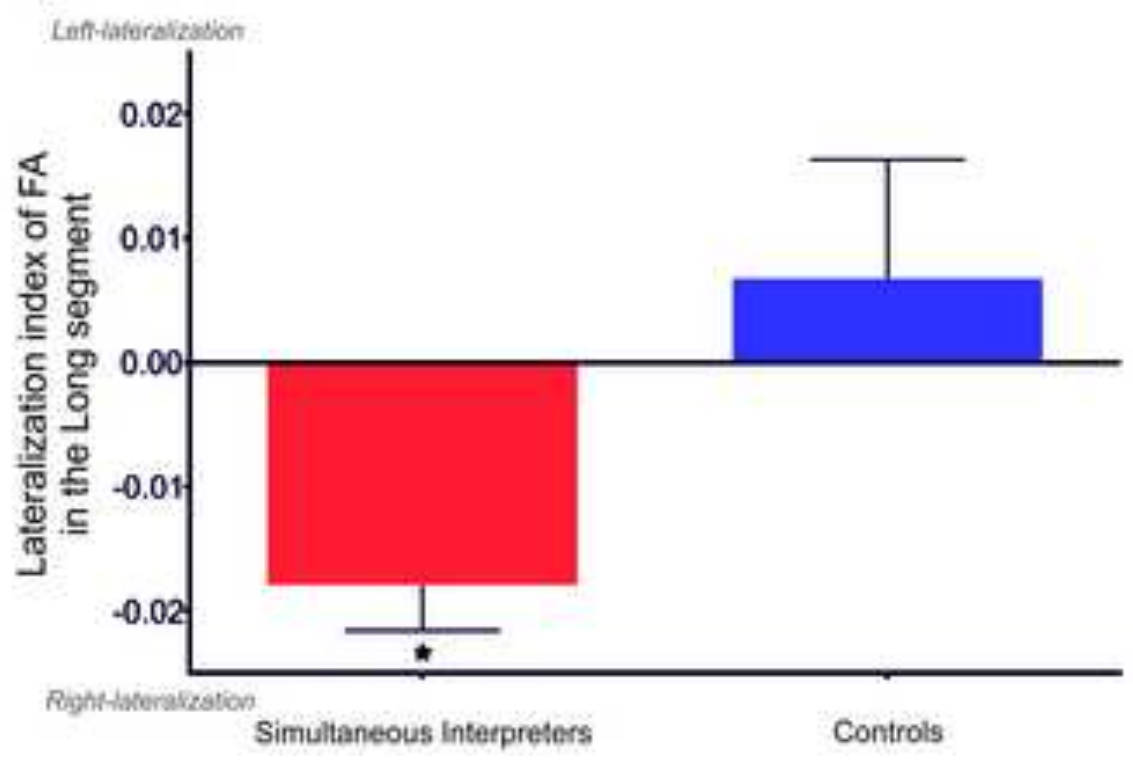

B)

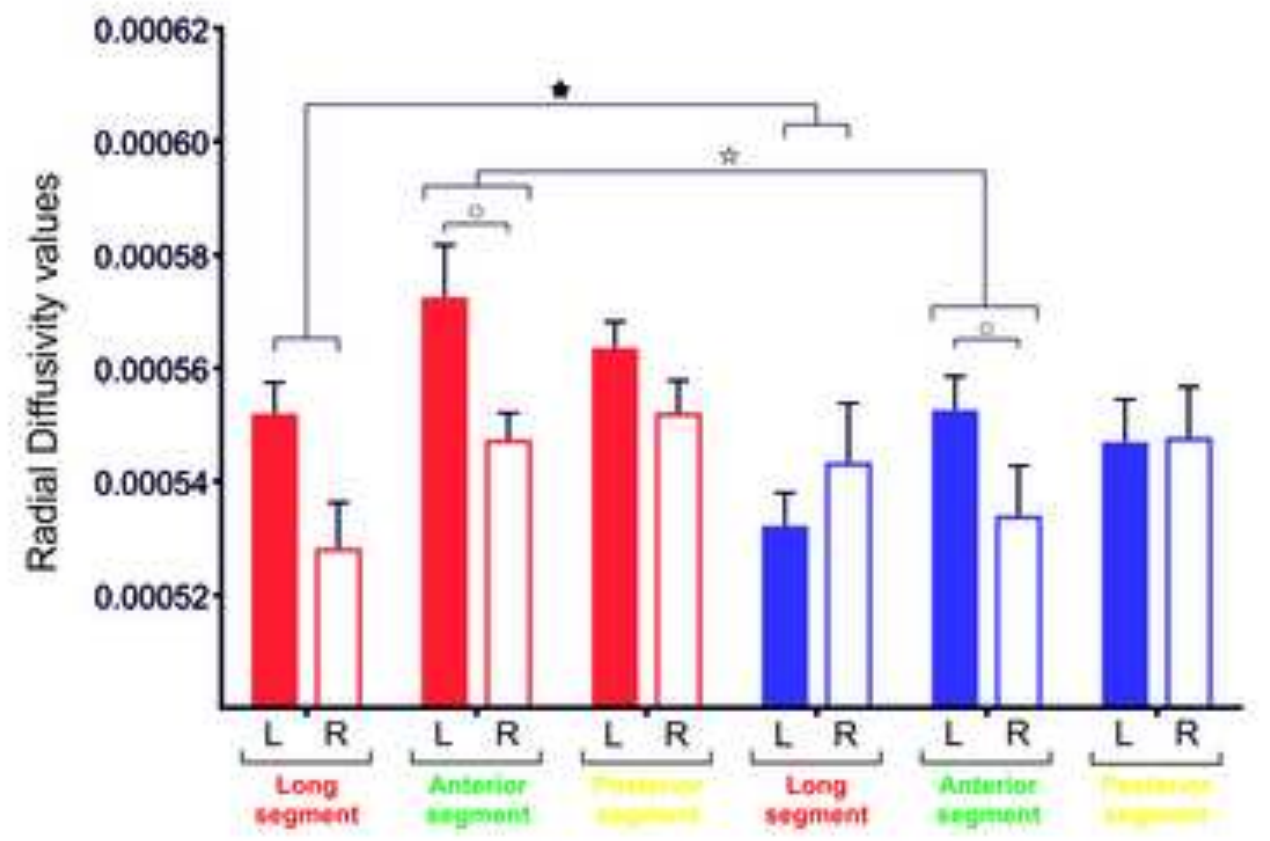

D)

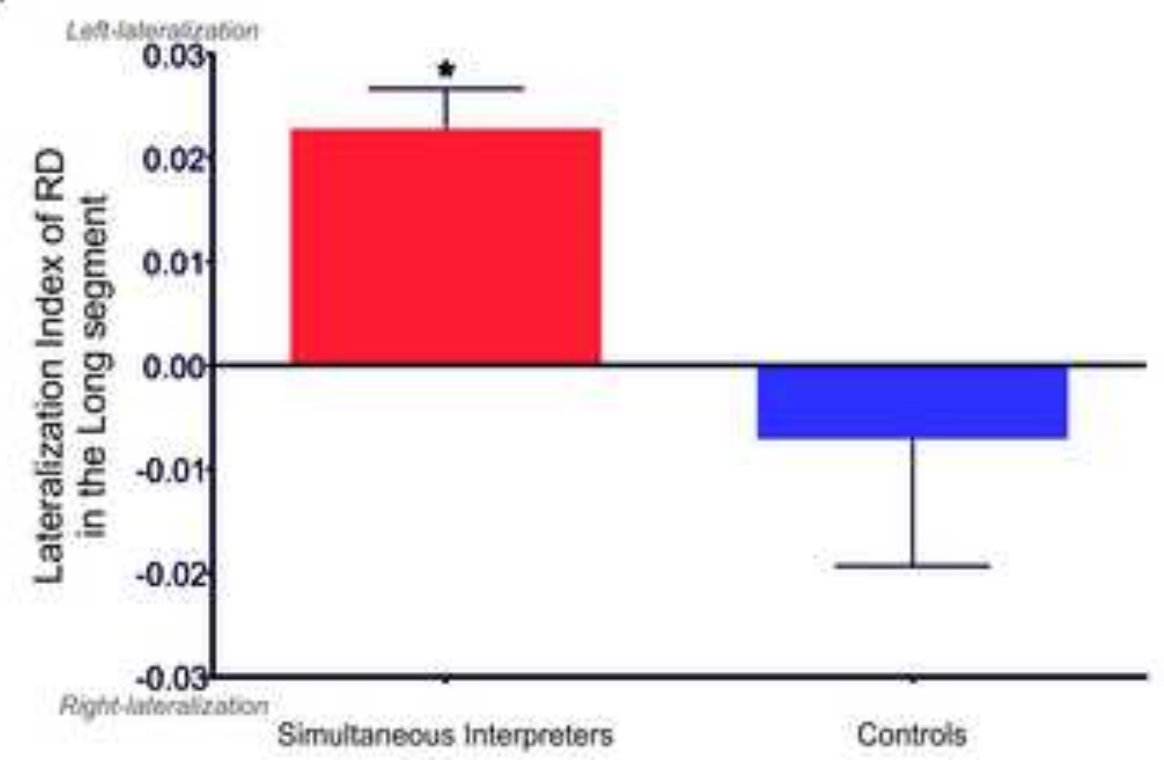




\section{Click here to download high resolution image}
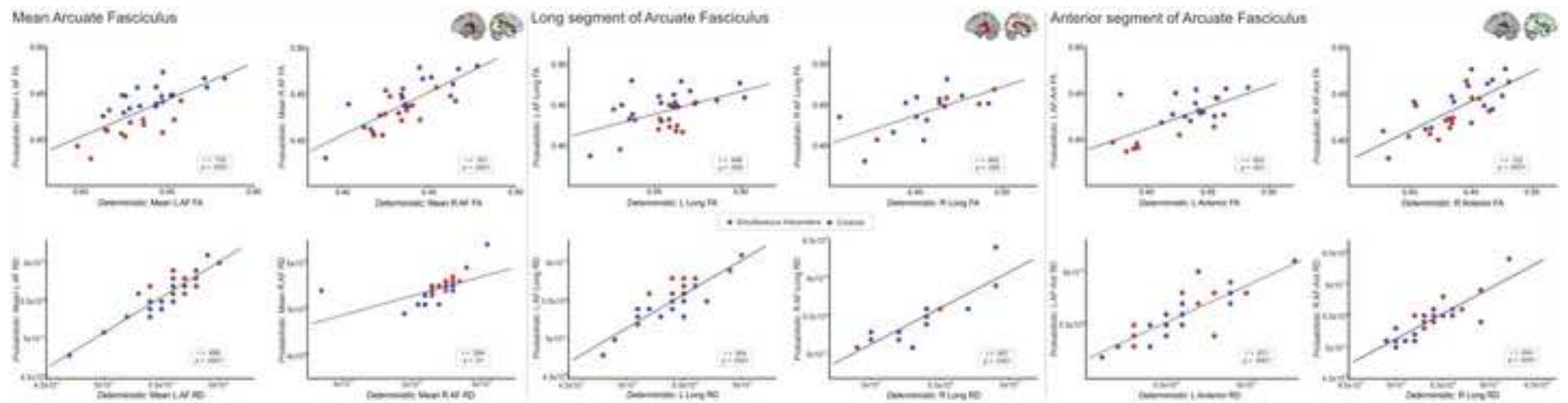